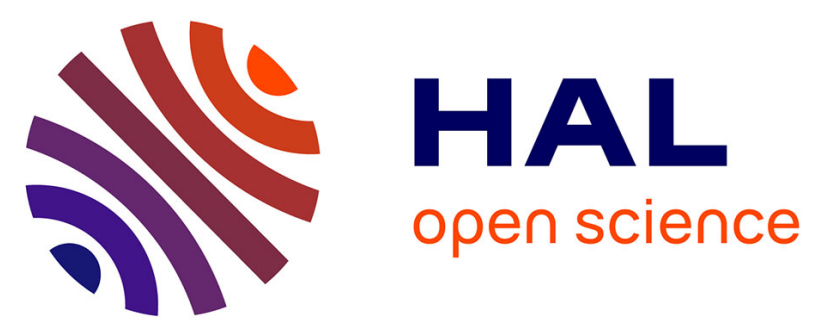

\title{
Cutting-edge multi-level analytical and structural characterization of antibody-drug conjugates: present and future
}

\author{
Alain Beck, Valentina D'atri, Anthony Ehkirch, Szabolcs Fekete, Oscar \\ Hernandez-Alba, Rabah Gahoual, Emmanuel Leize-Wagner, Yannis Francois, \\ Davy Guillarme, Sarah Cianférani
}

\section{To cite this version:}

Alain Beck, Valentina D'atri, Anthony Ehkirch, Szabolcs Fekete, Oscar Hernandez-Alba, et al. Cutting-edge multi-level analytical and structural characterization of antibody-drug conjugates: present and future. Expert Review of Proteomics, 2019, 16 (4), pp.337-362. 10.1080/14789450.2019.1578215 . hal-02272143

\section{HAL Id: hal-02272143 \\ https://hal.science/hal-02272143}

Submitted on 8 Oct 2020

HAL is a multi-disciplinary open access archive for the deposit and dissemination of scientific research documents, whether they are published or not. The documents may come from teaching and research institutions in France or abroad, or from public or private research centers.
L'archive ouverte pluridisciplinaire HAL, est destinée au dépôt et à la diffusion de documents scientifiques de niveau recherche, publiés ou non, émanant des établissements d'enseignement et de recherche français ou étrangers, des laboratoires publics ou privés. 
Cutting-edge multi-level analytical and structural characterization of antibody-drug conjugates: present and future

Alain Beck ${ }^{(1) \star}$, Valentina D’Atri(2), Anthony Ehkirch ${ }^{(3)}$, Szabolcs Fekete ${ }^{(2)}$, Oscar HernandezAlba $^{(3)}$, Rabah Gahoual(4), Emmanuel Leize-Wagner ${ }^{(4)}$, Yannis François ${ }^{(4)}$, Davy Guillarme ${ }^{(1)}$, Sarah Cianférani(3)*

(1) IRPF - Centre d'Immunologie Pierre-Fabre (CIPF), 74160 Saint-Julien-en-Genevois, France.

(2) School of Pharmaceutical Sciences, University of Geneva, University of Lausanne, CMU - Rue Michel-Servet, 1, 1206 Geneva, Switzerland.

(3) Laboratoire de Spectrométrie de Masse BioOrganique, IPHC UMR 7178, Université de Strasbourg, CNRS, 67000 Strasbourg, France.

(4) Laboratoire de Spectrométrie de Masse des Interactions et des Systèmes (LSMIS), UMR 7140, Université de Strasbourg, CNRS, France.

${ }^{*}$ Corresponding authors:

Alain BECK alain.beck@pierre-fabre.com

Sarah CIANFERANI sarah.cianferani@unistra.fr 


\section{ABSTRACT}

Introduction: The development and optimization of antibody drug conjugates (ADCs) rely on improving their analytical and bioanalytical characterization, by assessing critical quality attributes (CQAs). Among the CQAs, the glycoprofile, drug load distribution (DLD), the amount of unconjugated antibody (D0), the average drug-to-antibody ratio (DAR), the drug conjugation sites and the residual drug-linker and related product proportions (SMDs) in addition to high and low molecular weight species (H/LMWS) are the most important ones.

Areas covered: The analytical and structural toolbox for the characterization of $1^{\text {st }}, 2^{\mathrm{d}}$ and $3^{\mathrm{d}}$ generation ADCs was significantly extended in the last 3 years. Here, we reviewed state-ofthe-art techniques, such as liquid chromatography, high resolution native and ion mobility mass spectrometry, multidimensional LC and capillary electrophoresis hyphenated to mass spectrometry, reported mainly since 2016 .

Expert commentary: These emerging techniques allow a deep insight into important CQAs that are related to ADC Chemistry Manufacturing and Control (CMC) as well as an improved understanding of in vitro and in vivo ADC biotransformations. This knowledge and the development of quantitative bioanalytical assays will continue to contribute to earlydevelopability assessment for the optimization of all the ADC components (i.e., antibody, drug, and linker) and help to bring next-generation candidate ADC into the clinic and hopefully to the market.

\section{KEYWORDS}

Brentuximab vedotin, trastuzumab emtansine, gemtuzumab ozogamicin, inotuzumab ozogamicin, 3G-ADCs, small-protein fragment-DCs. 


\section{Introduction}

Antibody-drug conjugates (ADCs) are one of the fastest growing classes of oncology therapeutics. After half a century of research, the FDA and EMA approval of brentuximab vedotin (Adcetris, BV, 2011, systemic anaplastic large cell lymphoma), trastuzumab emtansine (Kadcyla, T-DM1, 2013, HER2-positive metastatic breast cancer) [1] and more recently inotuzumab ozogamicin (Besponsa, IO, B-cell precursor acute lymphoblastic leukemia [2]) and the re-approval of gemtuzumab ozogamicin (Mylotarg, GO, 2017, CD33-positive acute myeloid leukemia) [3] have paved the way for ongoing clinical trials that include 80 further ADC candidates $[4,5]$. The recent reapplication and FDA approval of GO were based on increased understanding of the ADC dosing, specific results of the pivotal clinical study and extensive additional clinical experience with the drug. GO originally received accelerated approval for treatment of older patients with relapsed CD33-positive AML in 2000 [6], but it was withdrawn from the market in 2010, when the confirmatory trial failed to demonstrate clinical benefit among safety concerns, such as a higher rate of induction fatalities on the GO combination arm compared with chemotherapy alone [7].

In addition, nine ADCs are in late clinical development (Biologic Licence Application (BLA), phase III or pivotal phase II, Table 1 [8,9]). These ADCs are all based on clgG1 or hlgG1 antibodies and directed against the following antigen targets (i.e., CD19, CD79b, DLL3, EGFRvIII, Folate R1, HER2+, Nectin 4, TROP2) overexpressed in tumors. Different linkers, payloads (i.e., auristatin, duocarmicin, irinotecan, exatecan, maytansinoid and pyrrolobenzodiazepines) and various conjugation chemistries have been used. Most of them are based on stochastic conjugation to hinge cysteine residues after mild reduction or on surface-exposed lysine residues, resulting in different DLDs and DARs (from 2 to 8). Seven of them are investigated in solid tumors and two in hemato-oncology.

Table 1: ADCs to watch in 2019

\begin{tabular}{llll} 
Company & ADC (payload) & Isot. Target & Stage indication \\
\hline Immunomedics & sacituzumab govitecan (IRI)* & IgG1 TROP-2 & BLA triple-neg. breast cancer \\
Daiichi Sankyo & trastuzumab deruxtecan (EXA)* & IgG1 HER2+ & Ph 3 breast cancer vs T-DM1 (HER2+++) \\
Synthon & trastuzumab duocarmazine (DUO)* & IgG1 HER2+ & Ph 3 breast cancer vs T-DM1 (HER2+++) \\
ImmunoGen & mirvetuximab soravtansine (MAY)* & IgG1 Folate R1 & Ph 3 epithelial ovarian cancer \\
Roche & polatuzumab vedotin (AUR) & IgG1 CD79b & Ph 3 diffuse large B-cell lymph. \\
AbbVie & depatuxizumab mafodotin (AUR) & IgG1 EGFRvIII & Ph 3 glioblastoma \\
Seattle Genetics & enfortumab vedotin (AUR)* & IgG1 Nectin 4 & Piv. Ph 2 urothelial cancer \\
AbbVie & rovalpituzumab tesirine (PBD) & IgG1 DLL3 & Ph 3 small cell lung cancer \\
ADC-Therapeutics & loncastuximab tesirine (PBD) & IgG1 CD19 & Piv. Ph 2 Diffuse Large B-Cell Lymphoma
\end{tabular}

+ 2 phase III failures (Seagen and Agensys)

*6 have FDA breakthrough or fast track designation or priority review 
Priority review for sacituzumab govitecan in $\operatorname{mTNBC}(\mathrm{Jul} 23,2018)$

Breakthrough therapy designation to trastuzumab deruxtecan for HER2+ MBC (Aug 30, 2017)

Fast track designation to trastuzumab duocarmazine for HER2+ MBC (Jan 28, 2018)

Fast track designation to mirvetuximab soravtansine for Pt-Resistant Ovarian Cancer (Jun 18, 2018)

Breakthrough therapy designation to polatuzumab vedotin + BR for lymphomas (Dec 12, 2017)

Breakthrough therapy designation to enfortumab vedotin for Metastatic Urothelial Cancer (Mar 28, 2018)

No $3 G$ site-specific conjugates

On the structural side, ADCs are much more complex than unconjugated mAbs, as the heterogeneity of the conjugates adds to the inherent microvariability of the biomolecules such as glycosylation [10]. The development and optimization of ADCs rely on improving their analytical and bioanalytical characterization, by assessing multiple critical quality attributes (CQAs) [11], namely the glycoprofile, the distribution and position of the drug (DLD), the amount of naked antibody, the average drug-to-antibody ratio (DAR) [12], the conjugation sites (surface exposed lysine, reduced hinge cysteine or engineered residues) [13-15], and the residual drug-linker and related product proportions (SMDs) [16].

The limited success of first-generation ADCs (1G-ADCs) [17] provides strategies to bring second-generation ADCs to the market (2G-ADCs), which have higher levels of cytotoxic drug conjugation, lower levels of unconjugated antibodies and more-stable linkers between the drug and the antibody. Furthermore, lessons learned during the past decade are now being used in the development of third-generation ADCs (3G-ADCs) with extended therapeutic windows [4]. Because of the hybrid nature of ADCs, product quality attributes (pQAs) for both the biological component [18] and the small-molecule components must be simultaneously considered [1921]. In addition, early-developability assessment requires well established but also state-ofthe-art analytical and structural techniques [22], such as native and ion mobility mass spectrometry, but also modern to multidimensional liquid chromatography and capillary electrophoresis methods hyphenated to mass spectrometry. As reviewed here, in the last 3 years, these emerging techniques [23,24] allowed a deep insight into important structural features that are related to ADC function and safety as well as an understanding of ADC biotransformation in vivo [25]. Based on these structure-function relationships data and by an iterative process, the $3 \mathrm{G}-\mathrm{ADC}$ s have now reached early and late clinical development stages. 


\section{State-of-the-art chromatographic methods}

\subsection{Size exclusion chromatography (SEC): native and organic}

Size-exclusion chromatography (SEC) is the reference technique for the qualitative and quantitative evaluation of protein aggregates (size variants). The main advantage of SEC is the mild chromatographic conditions that allow the analysis of therapeutic proteins with minimal influence on their conformational structure and local environment. Therapeutic protein aggregates ranging from dimer to visible particles have been recognized for their potential to elicit immune responses $[26,27]$. Because of these safety risks, the industry and regulatory authorities consider aggregation as a CQA [28]. SEC separates proteins into three major species: (i) high molecular weight species (HMWS), (ii) main peak (predominantly the monomeric form), and (iii) low molecular weight species (LMWS) $[29,30]$. Compounds are separated based on the difference between their hydrodynamic volumes, which results in different residence times that solutes spend in the internal pores of the stationary phase. Therefore, SEC mechanism is considered as an entropy-controlled process. Ideally, physicochemical interactions (adsorption) do not occur between the protein and stationary phase. Inert conditions need to be maintained during the analysis to avoid non-specific hydrophobic or electrostatic interactions (causing elution shift, peak distortion or poor recovery).

Non-denaturing aqueous buffers (e.g., phosphate) of medium ionic strength (containing $\mathrm{NaCl}$ or $\mathrm{KCl}$ ) are commonly applied to minimize non-desired electrostatic interactions with the surface of the stationary phase [31]. When analyzing antibody-related products such as ADCs, electrostatic interactions are often observed. A systematic evaluation of the salt additives on measured amounts of mAbs and ADCs evidenced their major role in aggregation assessment [32]. As an example, a significant change of aggregate level has been observed with a mobile phase containing $100 \mathrm{mM}$ sodium phosphate dibasic and $200 \mathrm{mM} \mathrm{NaCl}$, while no aggregates were observed without $\mathrm{NaCl}$ in the mobile phase. Despite the monomeric protein did not show important adsorption onto the stationary phase, enhanced secondary electrostatic interactions were suspected with the dimeric forms, due to its higher total number of positive charges and a conformational state which could increase the accessibility of protonated amino acids residues to the resin. In addition, when working with potassium-based mobile phases instead of sodium-based ones, better aggregate recovery was found. Therefore, potassium-based salts should be preferred over sodium salts, to reduce possible secondary interactions between the dimeric forms of mAbs/ADCs and SEC phases as well as to lower protein-salt interactions.

Hydrophobic interactions may also occur with hydrophobic species (e.g., with ADCs containing hydrophobic linkers and drugs). Therefore, often poor peak shape (large tailing) and 
incomplete resolution of aggregates from the monomeric conjugate are observed [33]. For such cases, the addition of an organic modifier to the mobile phase (i.e., methanol, isopropanol, acetonitrile, propylene glycol or DMSO) can be successfully used to overcome non-desired hydrophobic interactions [34,35]. Generally, less amount of protic solvent is required to properly decrease hydrophobic interactions compared to aprotic solvents. However, it is often compound and stationary phase dependent. It worth mentioning that viscous solvents (e.g., isopropanol) can drastically increase the column pressure and can lead to additional pressure induced artifacts.

SEC has also been used in combination with MS for analyzing the composition of ADCs. It can be performed either in non-denaturing or in denaturing conditions. Non-denaturing SEC conditions can be maintained by using volatile buffers (e.g., ammonium acetate, ammonium formate). However, a recent study showed that volatile buffers work well only for acidic mAbs and related compounds. Proteins with basic character eluted in broad peaks and with inappropriate recovery [36]. Using a mixture of water and an organic solvent as the mobile phase is often referred to as "organic size-exclusion chromatography" (O-SEC) and known as denaturing SEC [22]. In a recent study, denaturing O-SEC has been used to examine the extent of drug conjugation for both intact and de-N-glycosylated ADCs [22]. The accurate mass information for intact ADC served to confirm the integrity of the primary structure following conjugation, as well as identifying the major and minor product conjugate forms. Mobile phases used for the introduction of ADCs into the MS contained both organic (acetonitrile) and acidic (TFA) modifiers that result in ADC denaturation. Because the inter-chain disulfide bonds of conventional Cys-conjugated ADCs are partially reduced prior to conjugation, many of the resulting conjugated isoforms were detected in dissociated forms under denaturing O-SECMS conditions. Using SEC coupled to a multi-angle light scattering (MALS) detector in conjunction with UV absorption and the refractive index has also been reported as an alternative method for DAR determination. Recently SEC has been used for MAM analysis of monomer and dimer of Broadly Neutralizing HIV-1 Antibodies (bnAbs) [37]. Enriched dimer and monomer fractions were identified by peptide mapping (RPLC-MS). SEC has also been applied in multidimensional separations as discussed in section 5 and to non-denaturing MS, as detailed in section 4 .

The current trend in SEC of biopharmaceuticals is to use columns packed with sub-3 $\mu \mathrm{m}$ particles, making possible to perform ultra-high performance size-exclusion (SE-UHPLC or UHP-SEC) separations within 5-10 min [38,39]. UHP-SEC columns perform two or three times lower plate heights compared to conventional 5-10 $\mu \mathrm{m}$ SEC packing. It is also worth mentioning that state-of-the-art UHP-SEC columns of $150 \times 4.6 \mathrm{~mm}$ (which become popular today) can only be operated on fully optimized UHPLC systems possessing very low extra- 
column volume and variance [40]. Indeed, a significant loss in efficiency is expected not only with conventional HPLC systems but also with not-optimized UHPLC systems too (due to the very low retention of the compounds, $-1<k<0$ and related small column peak variance).

The need for determining aggregates in ADC products motivated column providers to develop more inert stationary phases. The historically applied mobile phase conditions have also been re-evaluated in the past few years to perform the analysis of highly hydrophobic ADC products. Working with such state-of-the-art conditions enabled the unbiased determination of HMWs in ADC formulations.

\subsection{Hydrophobic interaction chromatography (HIC)}

Hydrophobic interaction chromatography $(\mathrm{HIC})$ retains and separates the sample components according to their relative hydrophobicity using mild conditions (e.g., physiological pH conditions, ambient mobile phase temperature and no or moderate amount of organic solvents) which help to preserve the native-like conformation of protein species. Thanks to the applied mild conditions, non-covalent protein assemblies - such as cysteine conjugated ADCs - preserve their native conformation and do not dissociate into subunits (in contrast with RPLC). HIC then can separate individually loaded variants, while maintaining their Y-shaped mAbstructure and thus enabling the evaluation of the DLD and the calculation of average DAR $\left(D A R_{a v}\right)[41]$. Therefore, HIC is still considered as the reference technique for the detailed DAR analysis of cysteine conjugated ADCs (Cys-ADC, like BV). Nevertheless, HIC does not bring much for the analysis of lysine-linked (Lys-ADC) and site-specific ADCs. Indeed, due to its limited kinetic performance, conjugated and unconjugated species can only be resolved partially. Figure 1 shows examples on the application of HIC for the three different types of ADCs.

HIC separations are performed by running an inverse salt gradient on hydrophobic stationary phase. The stationary phase is less hydrophobic compared to RP phases, typically contains butyl, ether or alkylamide modifications [42]. The retention is based on protein salting-out. Proteins are adsorbed in the presence of high salt concentration and are released and eluted by decreasing the ionic strength. Historically, 1.5-2 $\mathrm{M}$ ammonium-sulfate aqueous solution is mostly used as mobile phase A, but it is important to keep in mind that various salts (sodium chloride, sodium acetate, ammonium acetate...) can be applied and can provide different selectivities. The influence of salts on hydrophobic interactions follows the lyotropic (Hoffmeister) series for the precipitation of proteins from aqueous solutions [42]. The retention in $\mathrm{HIC}$ is often increased with temperature, and this effect has been attributed to the enhanced magnitude of hydrophobic interactions resulting from temperature-induced conformational changes of proteins or to the concomitant increase in the hydrophobic contact area upon 
binding to the stationary phase. Typically, HIC separations are performed between 20 and 40 ${ }^{\circ} \mathrm{C}$.

It also worth mentioning that a small proportion of organic modifiers is often used as mobile phase additive for mAbs and ADCs. However, the effect of organic modifier on the separation is still not well understood [43]. Some studies justify the use of organic modifier with an increased resolution of sample components or attenuation of binding to the stationary phase of the most hydrophobic species. The use of either isopropanol (IPA) and/or acetonitrile (ACN) in a concentration range of $5-25 \%$ appear in the literature. Chen et al. reported the use of $50 \%$ ACN in mobile phase B in a hybrid form of RPLC and HIC separation, followed by direct LCMS analysis of model proteins [44]. Rodriguez-Aller et al. reported that the use of IPA in the mobile phase could be a parameter for tuning selectivity of mAb and ADC separation. In the case of ADC, IPA decreased the retention of the unconjugated host mAb and DAR2, but surprisingly increased the retention of DAR 4-8 species. Moreover, incomplete elution of higher DARs was observed above a given amount of IPA. This was possibly due to effects of $\mathrm{pH}$ shift, conformational changes, or increased salting out effect in the presence of high amounts of IPA (protic solvent) [41]. To conclude the presence of organic solvents, their use can evidently lead to the denaturation of protein biopharmaceuticals. Protic solvent (IPA) was found to be a more favorable solvent than aprotic one (ACN), to limit denaturation, but its proportion should remain reasonable (up to 10-15\%) [43]. Organic additives can modify the "apparent" average DAR value too, due to the improved recovery of the most hydrophobic species (DAR6 and DAR8 particularly) in the presence of IPA or ACN. Thus, special care should be taken when determining the average DAR value of cysteine linked ADCs.

An interesting concept was suggested recently for the HIC gradient optimization of cysteine linked BV. In this study, the DAR species were considered as members of homologs series [45]. It is known that when separating homologous series of compounds, non-linear (concave) gradients can provide better selectivity than linear gradients. It is due to the fact that in the case of homologs, the selectivity decreases as the homolog number increases. For homologs with low numbers of functional groups, one additional functional group can increase drastically the strength of interaction between the solute and the stationary phase. Conversely, for homologs possessing a high number of functional groups, one additional functional group will increase the strength of their interaction only to a small extent. Therefore, unequal peak distribution is often observed in the chromatograms due to the gradually decreasing molecular difference among the species possessing a higher position in the series. As an example, the difference in hydrophobicity of DAR0 and DAR2 species of a cysteine conjugated ADC is significantly larger than of the DAR6 and DAR8 species (as shown in the blue chromatogram of Figure 1a). For the above-discussed reasons, a logarithmic gradient profile was proposed 
to increase selectivity between high DAR species and decrease the "non-useful" elution space between the low DAR species. It has been derived that the logarithmic gradient can provide theoretically the best selectivity between homologs peaks (equidistant band spacing is expected). In practice, the logarithmic-like gradients can be performed by approaching the logarithmic shape with a multilinear gradient. This way, the retention of solutes can be predicted for logarithmic-like gradient profiles on the basis of only two linear scouting gradients. Another important advantage of the logarithmic profile is its peak focusing effect for the less retained DAR0 species. This is particularly useful because the concentration of DAR0 is often low (the naked $\mathrm{mAb}$ is considered as an impurity of $A D C$ ). By utilizing the peak focusing effect, the quantitation limit of DAR0 can thus be improved.

Clearly, HIC regained its interest in the past few years, mostly thanks to ADC developments. Several new columns and concepts have been developed and are now available. The retention behavior of large biomolecules is better understood. There is no doubt that $\mathrm{HIC}$ will remain the standard method for cysteine linked ADCs, to determine DAR distribution. Further developments in HIC column technology are also expected to improve recovery of hydrophobic loaded species and increase separation efficiency.

\subsection{Ion exchange chromatography (IEX)}

Ion-exchange chromatography (IEX) is a historical and non-denaturing technique widely used for the characterization of charge variants of therapeutic proteins. IEX is now routinely applied for $\mathrm{mAb}$ characterization, especially for the separation of acidic and basic variants, related to post-translational modifications. [46] IEX analysis of mAbs is often performed at either intact or sub-unit level (Fc and Fab analysis) [47]. IEX is also often used for studying mAb stability under accelerated storage conditions [48].

Cation exchange chromatography (CEX) is carried out by applying a shallow gradient of increasing salt concentration (e.g., sodium chloride) at constant $\mathrm{pH}$. In addition to choosing the appropriate $\mathrm{pH}$ of the starting buffer, its ionic strength (salt concentration) should be kept low. The proteins are then eluted by increasing the salt concentration to increase the competition between the buffer ions and proteins for charged groups on the IEX resin. An alternative approach for the separation of charge variants consists in applying a $\mathrm{pH}$ gradient (using specific buffers), whilst keeping constant the ionic strength. Both salt- and $\mathrm{pH}$ gradient based separations are used to perform multi-mAb (generic or platform) methods.

Depending on the changes in the chemical and physical characteristics of an antibody upon conjugation with a small molecule drug and the conjugation site, methods that can be applied to the parent antibody may not be applicable to the ADC [26]. Conventional IEX is indeed not adapted to cysteine conjugated ADCs, as the presence of linked cytotoxic drugs increases the 
hydrophobicity and might not impact the net charge of the antibody relative to the unconjugated mAb. This may result in poor separation quality under IEX conditions.

On the contrary, the attachment of an uncharged linker and drug through lysine residues decreases the net positive charge by one for each bound drug-linker. Therefore, separation based on the charge may result in chromatographic profiles that characterize the drug load, rather than the underlying mAb. Figure 1 shows examples on the application of CEX for TDM1 and GO. Partially resolved peaks can be expected at intact level analysis.

Similarly, if charged payloads [49] are conjugated on the antibody - even through cysteine residues - then the use of IEX would make sense. Probably, its separation quality would be poor, and RPLC or HIC could better separate the DAR species. In this case, IEX could provide some information about the payload or at least should partially separate unconjugated and conjugated species. There are only a few published reports of charge-based analysis applied to ADCs. Multiple charge variant peaks were observed during CEX analysis of several different engineered ThiomAb conjugations [50]. This charge heterogeneity was due to cysteinylation and/or glutathionylation at the engineered and unpaired cysteines through disulfide bonds formed during the cell culture process. In another study, anion exchange chromatography (AEX) was used to analyze Fc-conjugates (fluorescent probes were conjugated through lysine) [51]. The AEX method enabled to determine the amount of unconjugated versus conjugated proteins. However, for species with DAR > 2, it was difficult to resolve the individual species.

Due to the trend to develop ADCs with charged payloads, or attach uncharged payloads through lysine, the interest for IEX will probably increase in the future. Instead of analyzing the $A D C$ at the intact level, their analysis at subunit level can improve the separation quality. More efficient IEX columns are also expected to be developed in the close future.

\subsection{Reversed-phase liquid chromatography (RPLC)}

Thanks to its inherent robustness, high resolution, and versatility, reversed-phase liquid chromatography (RPLC) is widely used for the analysis of therapeutic proteins. The clear advantage of RPLC over HIC is its inherent compatibility with MS, due to the volatile nature of the mobile phase. However, it is important to keep in mind that: i) elevated mobile phase temperature $\left(60-90^{\circ} \mathrm{C}\right)$ is a prerequisite for successful RPLC analysis of ADCs, to limit oncolumn adsorption [52] and memory effects [23], ii) ion pairing agents $(0.05-0.1 \%$ TFA) has to be systematically added to the mobile phase to avoid peak broadening and tailing of multiply charged solutes [53], iii) organic solvent (20 - 50\% of methanol or acetonitrile) is mandatory to elute ADC samples. For the specific case of ADC, RPLC has been used for the assessment of (DAR $\mathrm{av}_{\mathrm{av}}$, determination of drug load distribution, evaluation of unconjugated antibody amount, as well as free drug-linker quantitation [26,54]. 
For Cys-ADC, RPLC can be considered as a suitable alternative to HIC for the determination of $D R_{a v}$. Such an application is illustrated with the representative chromatograms of BV in Figure 1a. However, the harsh RPLC conditions (i.e., elevated temperature, acidic conditions and use of an organic solvent) does not allow maintaining the physiological-like Y-shape structure of the antibody through only electrostatic and hydrogen bond interactions (there are no more covalent bonds between chains in Cys-ADC). Indeed, even a small amount of organic solvent has proven to be disruptive and could lead to antibody dissociation into the light and heavy chains under RPLC conditions [24]. Therefore, the successful analysis of these ADCs cannot be performed under native conditions, and this sample must be treated with reductants such as dithiothreitol (DTT) to have information on the DLD on the light and heavy chains. In this case, the DAR species related to the light chains ( $L, L 1)$ and heavy chains $(H, H 1, H 2, H 3)$ of reduced Cys-ADC can be separated, and $D R_{a v}$ easily estimated using peak area percentages and simple equations, as previously reported. Among the different species that need to be separated, it is often quite challenging to resolve the light chain with one payload (L1) from the unconjugated heavy chain $(H)$, since these two species have very close lipophilicities (RPLC separation is mainly driven by hydrophobicity). However, the additional selectivity provided by a high coverage phenyl bonded phase (related to steric and $\pi-\pi$ interactions), can seriously help to better discriminate these protein species [55,56], as recently shown. Besides the use of polyphenyl column, a general approach to find the best possible separation in RPLC is to use retention modeling softwares. Indeed, by simultaneously modeling the effect of temperature (in the range $60-90^{\circ} \mathrm{C}$ ), gradient steepness, and possibly mobile phase ternary composition $\left(\mathrm{MeOH} / \mathrm{ACN} / \mathrm{H}_{2} \mathrm{O}\right)$, optimal conditions of separation can be rapidly determined (2-3 working days), based on a limited number of initial experiments [57] (between 4 and 12, depending on the number of parameters initially considered). In addition, the average error of retention time prediction is typically lower than $0.5 \%$. It is finally also important to notice that the $\mathrm{DAR}_{\mathrm{av}}$ can also be successfully measured based on subunit specific analysis in which ADCs are enzymatically fragmented with IdeS and then chemically reduced to get Fc/2, LC and Fd fragments, as shown in Figure 1a. The main issue related to the measurement of $D A R_{a v}$ from chromatographic peaks is related to the incomplete recovery of loaded species and therefore mobile phase temperature need to be sufficiently high $\left(80-90^{\circ} \mathrm{C}\right.$ ideally). Determination of $\mathrm{DAR}_{\mathrm{av}}$ is further complicated by the limited chromatographic resolution, related to the heterogeneous nature of ADC (presence of isomers, PTMs...), the fact that analysis time should not be longer than 20 minutes, to avoid thermal on-column degradation and some possible solubility issues for the most hydrophobic DAR species.

For Lys-ADCs, the integrity of the ADC sample is maintained even under the harsh conditions employed in RPLC, since the inter-chain disulfide bridges are kept. However, as illustrated in 
Figure 1b with T-DM1 and GO, the resolving power of RPLC is too low to discriminate the various DAR species of Lys-ADC, and a single broad peak is observed. This was obviously attributed to the high heterogeneity of Lys-ADC and the fact that mAbs have more than 60 surface-exposed lysine residues randomly available for the conjugation [58]. The chromatograms shown in Figure 1b confirms that RPLC cannot be considered as a powerful technique for the analytical characterization of Lys-ADC at intact level.

For site-specific ADC, the payloads are attached to defined positions that are suitable for drug conjugation. In addition, these products have a theoretically near-uniform stoichiometry of cytotoxic molecules attached per antibody molecule, without disruption of inter-chain disulfide bonds. Even if HIC is still considered in the industry as the method of choice for the analysis of intact site-specific ADCs under non-denaturing conditions, RPLC can also be used as the sample complexity is significantly simplified, making intact DAR characterization by RPLC feasible. Xu et al. [59] demonstrated the use of RPLC for DAR characterization and drug distribution of both reduced and intact site-specific non-natural amino acid-based ADC. They showed the possible discrimination of unconjugated species (DAR0), one drug species (DAR1) and two drugs species (DAR2) at the intact ADC level of analysis (without reduction). The identity of these different peaks was confirmed by MS, and $\mathrm{DAR}_{\mathrm{av}}$ was easily calculated by considering their corresponding peak areas. In this study, the authors have also shown that this approach provides slightly lower, but still comparable $D R_{a v}$ values in comparison to the reduced RPLC method. Recently, we have also confirmed the benefits of using RPLC vs. HIC for the analysis of site-specific ADCs at the intact level. As illustrated in Figure 1c, the proposed RPLC method allows i) separation of the unconjugated mAb from the ADC species and easy quantitation of DARO amount, ii) separation of the DAR species and calculation of the $\mathrm{DAR}_{\mathrm{av}}$ and DLD, iii) separation of some positional isomers of DAR species [60]. In addition, because of the inherent compatibility with MS, unambiguous identification of the peaks was performed thanks to the high mass accuracy of TOF-based instruments. Finally, mild conditions were employed in RPLC (mobile phase temperature of $60^{\circ} \mathrm{C}$ and $0.05 \%$ TFA as mobile phase additive $v s .80^{\circ} \mathrm{C}$ and $0.1 \%$ TFA in the study of $\mathrm{Xu}$ et al.), [59] thanks to the use of state-of-the-art RPLC column, thus limiting potential degradation of ADC species during their analysis. These results clearly demonstrate that the RPLC analysis of intact site-specific ADC can be considered as QC friendly and can serve as a release assay, since minimal sample preparation is needed, leading to a robust analysis.

Whatever the ADC type (i.e., Cys-ADC, Lys-ADC, and site-specific ADC), it is also important to determine and quantify the amount of small molecules impurities added into ADC products during manufacturing and storage. In this context, RPLC is widely used today to measure the free drug level and monitor in-situ small molecule drug stability, as RPLC is known to be highly 
accurate, sensitive and selective for small molecules compared to other techniques. Since the properties of $A D C$ and small molecule drugs are vastly different, free drugs can be easily separated from the ADC species by protein precipitation or solvent extraction. [26] As an alternative, RPLC can also be used in the second dimension of the 2D-LC setup, to perform free drug analysis without sample pretreatment, as discussed in section 5 .

One of the limitations of RPLC is that it relies on hydrophobicity differences among the species having various levels of conjugation. Therefore, it may not be suitable for monitoring drug conjugates with low hydrophobicity. In such a case, HILIC can be an alternative solution, as described below.

\subsection{Hydrophilic interaction chromatography (HILIC)}

Hydrophilic interaction chromatography (HILIC) is a valuable orthogonal approach to RPLC since it allows the separation of hydrophilic variants of peptides and therapeutic proteins. Even though more generally applied for released glycans analysis, recently developed wide-pore HILIC phases paved the way into new interesting applications of this LC mode for biopharmaceutical analysis [61-63]. Specifically, in the first contribution by Periat et al. [61], HILIC separations applied to protein analysis (including insulin, interferon $\alpha-2 b$, and trastuzumab) were found to be highly orthogonal to RPLC, while keeping comparable kinetic performance, and providing the unique ability to resolve glycoforms at the protein level. However, some care should be dedicated to the experimental setup for obtaining a successful separation. In this regard, the authors highlighted the fact that wide-pore amide-bonded HILIC stationary phases should be preferred to maximize protein retention and resolution. Furthermore, and similarly to RPLC, the use of ion-pairing agents $(0.05-0.1 \%$ TFA) was required to ensure proper peak shape and resolution and more importantly to impart and control the acidic conditions of the mobile phase. In fact, these behaviors plus the ability of TFA to ion-pairing with the glycoprotein were crucial to avoid ionic interactions that would have been manifest in unpredictable and unstable retention times. Finally, the critical impact of the injection volume on the breakthrough effect was also discussed. In this context, it should be taken in mind that in HILIC separations the aqueous solutions have the highest eluotropic strength; therefore, sample injected in aqueous solutions could result in distorted peaks or even be eluted within the column void volume (breakthrough effects). For this reason, small injection volumes $(0.5-1 \mu \mathrm{L})$ were suggested, together with the addition of a fast initial ACNrich ramp at the beginning of the gradient.

In reason of the great potential of HILIC-MS, and applying the above-mentioned cautions, D'Atri et al. [63] used this approach for the characterization of the Cys-ADC BV. Interestingly, and although the complexity of the ADC, complementary and orthogonal set of information 
were obtained as compared to RPLC-MS analysis performed on IdeS digested and DTT reduced samples. Specifically, the characterization of the ADC subunits heterogeneity, arising from drug payloads, post-translational modifications (PTMs) and glycosylation pattern, was effectively assessed in a unique HILIC-MS analysis. The major advantage of the approach consisted in the first instance in obtaining the protein glycoforms chromatographically separated and thus allowing an easier mass deconvolution and identification and, eventually, a simplified way to perform a batch-to-batch comparison of the glycosylation pattern. Then, an additional advantage was represented by the fact that the most hydrophobic species, mainly consisting of the sub-units carrying the cytotoxic drug payload, eluted at the beginning of the gradient in HILIC mode. This peculiar behavior allowed their good recovery that could be a potential issue in RPLC (see also Figure 1a for a straight example), and the relative percentage of each sub-unit was found to well correlate in both LC methods. Furthermore, additional positional isomers of the Fd subunit were detectable at a chromatographic level only by HILIC mode, thus highlighting the cutting-edge potential of this separation mode compared to RPLC.

A generic method development approach was also recently proposed by Bobaly et al. [62] to analyze a wide range of recombinant $m A b s$ and the Cys-ADC BV. In this contribution, generic HILIC conditions were adapted for protein separation at subunit level, and further optimization of the method was performed on three selected samples (including BV) to maximize resolution. Specifically, recovery of ADC subunits and sample stability under the applied conditions were investigated, highlighting the fact that the proper recovery of sub-units carrying the cytotoxic drug payloads is crucial to avoid biased average DAR calculation. Namely, at least $80^{\circ} \mathrm{C}$ was found to be required for assuring $90 \%$ recovery of the species bearing the drug payloads.

To conclude, due to the increasing popularity of HILIC-MS for biopharmaceutical analysis, future applications in the field of ADC characterization could involve: i) Comparison of the glycosylation patterns between originator and biosimilar ADCs, as recently demonstrated for a large set of mAbs [64,65], ii) Characterization of product-related low molecular weight impurities, as recently demonstrated for a recombinant IgG1 mAb [66], and (iii) Interesting twodimensional LC-MS approach for separating and detecting both high and low abundant glycan species, as recently demonstrated through the analysis of three therapeutic mAbs [67]. 
a)

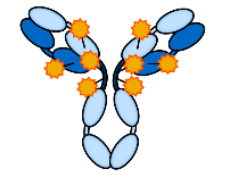

Cysteine conjugation

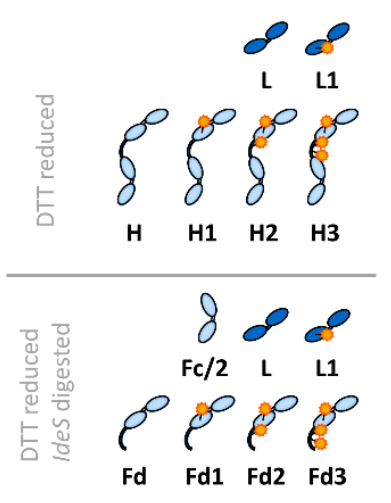

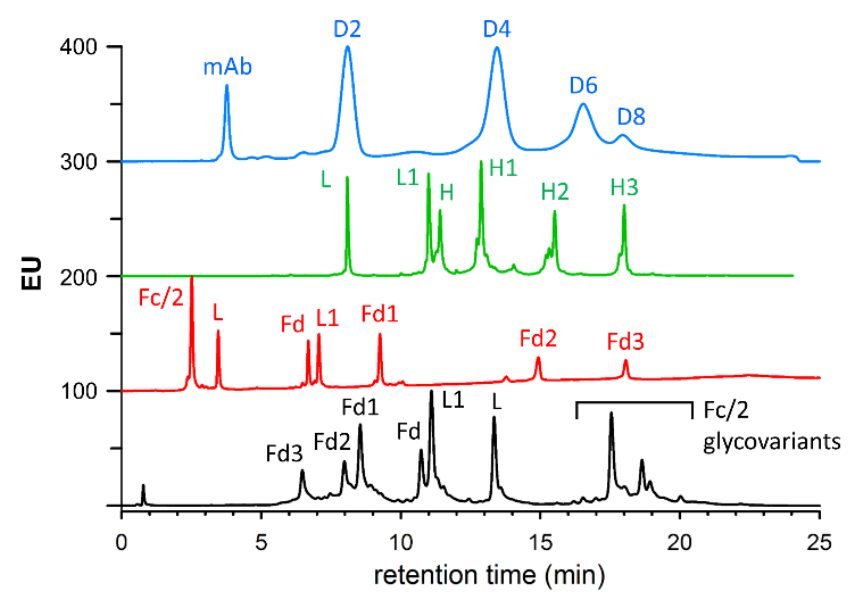

b)

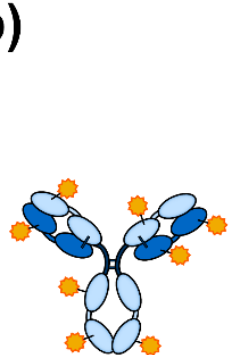

Lysine conjugation
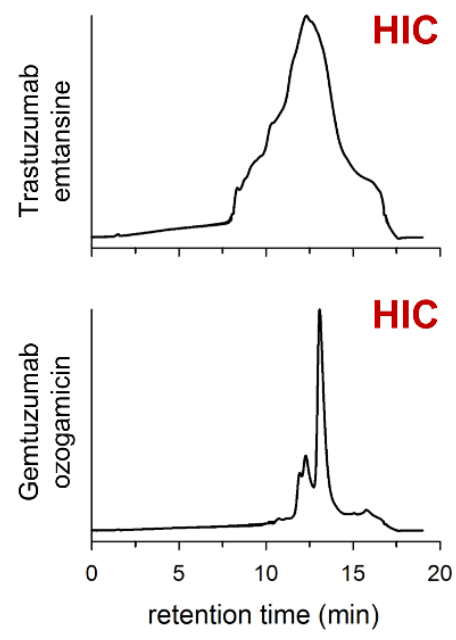

c)

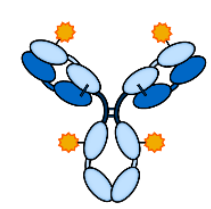

Site-specific conjugation
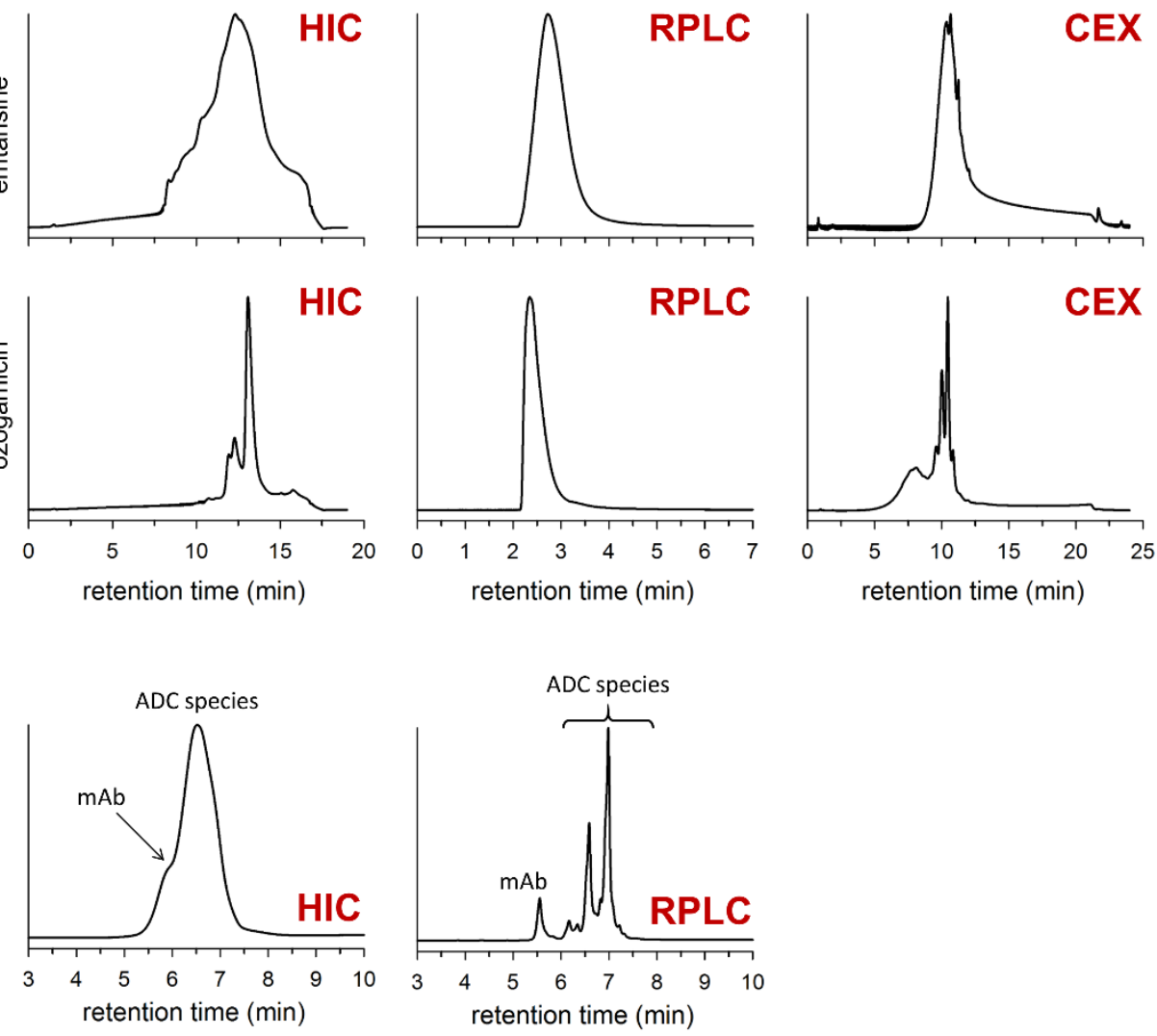

Figure 1. Chromatographic approaches applied to ADCs. a) Cys-linked ADC and its relative subunits upon DTT reduction or IdeS digestion and DTT reduction (panel on the left) analyzed by orthogonal chromatographic methods (panel on the right). The intact sample analyzed by HIC (blue line), DTT reduced sample analyzed by RPLC (green line) and IdeS digested and DTT reduced sample analyzed by RPLC (red line) and HILIC (black line), respectively. Numbers refer to the number of drug payloads linked to each specific species or subunits. Figure readapted from [54]. b) Representation of a Lys-linked ADC and HIC, RPLC and CEX chromatographic profiles obtained for trastuzumab emtansine (top panels) and gemtuzumab ozogamicin (bottom panels) analyzed at intact level. [Data from the authors' laboratory]. c) Representation of a site-specific conjugated ADC and comparison of HIC and RPLC chromatographic profiles obtained for an investigational sample analyzed at intact level. Figure readapted from [60]. 


\section{State-of-the-art electrophoretic methods}

Because of its simple instrumentation, miniaturized format and outstanding separation efficiency, capillary electrophoresis (CE) represents a powerful technique to characterize biomolecules [68,69]. CE possesses many advantages, including fast separation, small sample consumption and high resolution, and can be considered as a key complementary technique to chromatographic approaches for the analysis of protein biopharmaceuticals $[53,70,71]$. The different electrophoretic modes that can be employed, such as capillary gel electrophoresis (CE-SDS) [72], capillary isoelectric focusing (cIEF), imaged clEF (iclEF) [73] and capillary zone electrophoresis (CZE) [74-76], exhibit attractive opportunities for the characterization of ADCs at different levels, such as intact or reduced charge- or size-variants, isoforms, glyco-profiling and PTMs [26,70,71].

\subsection{Capillary Gel Electrophoresis (CE-SDS)}

Since the 90s, CE-SDS is recognized as an essential analytical technique in the biopharmaceutical industry for the characterization of mAbs [77-80], especially to determine the apparent molecular weight of proteins and assess the molecular size distribution and purity. CE-SDS is a charge-based separation mode which is quite orthogonal to SEC for the characterization of size variants, particularly to differentiate low molecular species in the range of $10-150 \mathrm{kDa}$. CE-SDS is a method based on the same mechanism as sodium dodecyl sulfate-polyacrylamide gel electrophoresis (SDS-PAGE). After the complete reaction between SDS and proteins, SDS-protein complexes with the same negative charge are formed allowing electrophoretic separation in a sieving medium only based on their hydrodynamic radius directly into the capillary. CE-SDS is classically coupled with UV or fluorescence detection.

Focusing on the characterization of ADCs, CE-SDS particularly can be used to provide critical information regarding the nature of aggregates and/or fragments following different modes of sample preparation, namely after reduction or not. However, while CE-SDS is a routine method for determining overall mAbs purity, this method has some limitations for the analysis of ADCs depending on the conjugation chemistry and the binding site (lysines, cysteines or glycans) $[24,26,33]$.

Concerning the interchain-cysteine modified ADCs, the conjugation of the cytotoxic drugs to antibodies is performed through cysteine sulfide groups which are activated by a partial reduction of interchain disulfide bonds. It means that some antibody chains are no longer covalently linked by intact disulfide bonds. The presence of SDS in the sample implies the formation of protein-SDS complexes and the dissociation of non-covalent interactions. This involves the dissociation of any antibody chains depending on the position and the number of cytotoxic drugs and then causes the presence of low molecular masses ADC fragments. The 
first study on CE-SDS analysis of interchain-cysteine modified ADCs corresponding to chimeric BR96 linked by anticancer drug doxorubicin (BR96-DOX) was published in 1995 by Liu and co-workers [81]. In the denatured state, six peaks were identified corresponding to the expected light chain, heavy chain, light-heavy chain, heavy-heavy chain, light-heavy-heavy chain and the unmodified antibody, with a predominance of the light chain, heavy chain, lightheavy chain species. Ten years later, Sun et al. reported similar results in the analysis of a cAC10-vc-MMAE immunoconjugate [82]. ADCs were analyzed under denaturing but nonreducing conditions on an automated silicon chip-based CE-SDS apparatus. CE-SDS combined with RPLC and HIC analysis demonstrated that treating CAC10 with reducing agents such as DTT caused preferential reduction of light-heavy chain disulfides, whereas reoxidation of fully reduced CAC10 interchain disulfides caused preferential reformation of lightheavy chain disulfides. Calculation of the positional isomers was performed within the isolated DAR fractions by associating the abundance of specific dissociation peaks in RPLC or CESDS with a specific isomer. The positional isomer distribution of the parent material could then be reconstructed from the information about the isolated DAR species. Based on this work, Le et al. used information about the abundance of different DAR species (HIC data) combined with CE-SDS dissociation of the entire, unfractionated sample to develop a mathematical approach allowing to determine the positional isomer distribution within a population of ADCs [83]. A survey of several interchain-cysteine modified ADCs based on the same lgG framework and small molecule drug (vc-MMAE) combination validated this approach by obtaining a similar distribution of isomers among all the molecules.

Concerning Lys-ADCs, CE-SDS profile looks totally different as compared to cysteineconjugated ADCs. Indeed, Lysine conjugation chemistry did not imply any partial reduction of interchain disulfide bonds meaning that antibody chains conserved covalently linkage properties by intact disulfide bonds. In 2016, Chen and co-workers published non-reducing CE-SDS studies of T-DM1 and the unconjugated mAb, trastuzumab. CE-SDS analysis gave a comparable profile with a prominent peak corresponding to the full-length antibody and minor peaks representing lower and higher molecular weight species [26]. The same year, another group described the in-depth structural characterization of T-DM1 and its biosimilar candidate using several analytical methods [84]. Characterization of size variants performed with the combination of SEC and CE-SDS showed similar results exhibiting predominant monomer contents (>95 \%) in T-DM1 and the biosimilar ADC sample indicating their similarity in the aspect of size heterogeneity. In 2018, Wagh et al. confirmed that non-reduced CE-SDS analysis usually shows a single prominent peak corresponding to full-length ADC in another Lys-ADC [24]. However, they demonstrated that reduced CE-SDS analysis usually shows prominent peaks of the light chain and heavy chains as well as minor peaks for non- 
glycosylated heavy chains and non-reducible species. Presented results demonstrated the possibility to separate light chains with 0 to 2 drugs molecules using reduced CE-SDS.

\subsection{Capillary Isoelectric Focusing (cIEF) and imaged cIEF (icIEF)}

Like electrophoretic gel modes for the analysis of therapeutic protein size variants, isoelectric focusing modes became these last decades reference methods for the analysis of mAb and ADC charged variants $[53,70,71]$. Due to their charge heterogeneity, the charge profile represents the unique "fingerprint" of mAbs or ADCs. The biopharmaceutical industry used some analytical techniques such as isoelectric focusing slab gel (IEF), cIEF and iclEF to characterize charge variants. Separation mechanism is based on the migration of proteins isoforms following their isoelectric point $(\mathrm{p} /)$ in an ampholytic $\mathrm{pH}$ gradient under the application of an electrical field. While IEF has been used in the last years to characterize the charge profile of ADCs, this method is however known as labor-intensive, time-consuming and not very reproducible. Adaptation of IEF to capillary format permits to reduce drawbacks and brings some advantages like ease of handling, automated procedure, short analysis time and improved resolution. In conventional cIEF, following the focusing step, electrophoretic or hydrodynamic mobilization is implemented to force the sample to move toward the UV detection. However, the mobilization step could have negative effects on the migration time and on the resolution, due to possible peak broadening. Because of these drawbacks, wholecolumn imaging iclEF has been established to achieve faster separation with greater resolution, better reproducibility, and reduced sample volume. Short capillary length $(5 \mathrm{~cm})$ combined to real-time monitoring of the focusing step allowing no mobilization step, lead to reduce analysis time and to perform high-throughput experiments. Those benefits allow for applications across entire pharmaceutical processes, from cell culture development and optimization to commercial quality control release and stability activities [85].

As CE-SDS, depending on the conjugation chemistry (nature of the drug-linker) and the binding sites (lysine, cysteine or glycan), isoelectric focusing methods may give significantly different information on electrophoretic profiles of ADCs [26,33]. The nature of the drug-linker, especially the charge that can or cannot be added to the ADC represents a major concern on the charge profile of the protein. For instance, $\mathrm{Ji}$ et al. described the characterization of thio-succinimide hydrolysis of vc-MMAE ADC using icIEF during the formulation development [86]. As uncharged vc-MMAE conjugation through sulfhydryl groups in the interchain-cysteine residues is known not to change the net charge of the ADCs, similar charge variant profiles were expected between the conjugated and the unconjugated mAb. However, overlay of iclEF profiles of vc-MMAE ADC, incubated at $\mathrm{pH} 9$ during 0 to 48 hours, showed an important heterogeneity in the acidic region due to various negative charges of the ADC gained from 
both deamidation and succinimide hydrolysis of the thio-succinimide linker. Each peak shift to the acidic region of the charge profiles represents a gain of one charge and then changes $\mathrm{p} /$ values. Based on this conclusion, an approach using weighted peak area and drug load has been utilized to quantify the thio-succinimide hydrolysis of ADCs [86]. Adem et al. described the same kind of results in the study of physical instability and the role of drug payload of vcMMAE ADC [87]. They represented acidic variants variation of ADCs after formulation in either low and high ionic strength buffer and storage at $40^{\circ} \mathrm{C}$ for up to 4 weeks. Despite significant differences in the aggregate formation of vc-MMAE ADC under high and low ionic strength buffer conditions, a similar increasing degree in acidic charge variant profiles were observed. More recently, Valliere-Douglas and co-workers demonstrated that freeze-dried mAbs and ADCs could be covalently modified with buffer and excipient molecules on the side chains of Glu, Asp, Thr, and Ser amino acids when subjected to temperature stress [88]. Two ADCs corresponding to an interchain cysteine modified vc-MMAE ADC and an engineered IgG1 with cysteine conjugated with a drug-linker molecule containing a pyrrolobenzodiazepine (PBD) dimer [89], were analyzed by iclEF. Significant changes were observed in the charge variant distribution of all ADCs at stress storage temperatures. For both ADCs, an increase in basic variant level was observed over time. The increase in the basic variants trended with increasing storage temperature [88].

Conjugation of the amino groups accessible to lysine residues to form amide bonds with uncharged linker moiety eliminates basic sites in the proteins and changes ADC p/s [90]. The first presented results concerning IEF gel electrophoresis analysis of GO, showed that most of the calicheamicin was on approximately half of the antibody while $45-65 \%$ of the product was a low conjugated fraction, essentially unconjugated antibody [33,91]. In 2010, Maeda et al. reported the development of a robust and highly reproducible conventional cIEF analysis of both intact and deglycosylated GO [92]. Three years later, Lin et al. described the determination of mAb-DM4 with an average DAR of 3.6 by iclEF [90]. $p /$ of the drug-loaded species shifted toward acidic variants from the unconjugated mAb due to the increase in the number of DM4 drugs conjugated. Charge variant profile exhibited nine peaks corresponding to proteins with zero to eight DM4 drugs per mAb. More recently, Luo et al. confirmed these results performing the structural characterization of a mAb-DM1 ADC using the combination of UV/vis spectroscopy, LC/TOF-MS and icIEF analytical methods [93]. iclEF showed that 0-8 DM1 drugs were conjugated to a mAb. Average DAR value (3.2) measured with iclEF was in good agreement with UV/vis and intact mass values (3.3 and 3.1 respectively). Moreover, drug load distribution was also characterized by icIEF and LC/TOF-MS. The DM1 distribution profiles obtained by both methods were similar assessing the icIEF as a powerful method to measure the homogeneity of ADC population. However, authors assumed that characterization of drug load distribution by iclEF could be very challenging because it cannot 
identify the antibody with linker only from the antibody with linker-DM1. Wagh et al. reported the same conclusion summarizing the fact that iclEF can be applied to measure the level of unconjugated antibody and drug load distribution, but it cannot distinguish between conjugates, process intermediates and impurities [24].

\subsection{Capillary Zone Electrophoresis (CZE)}

CZE represents the most straightforward mode of electrophoretic separation. CZE is commonly performed in aqueous electrolytes composed of a buffer, which enables to control the $\mathrm{pH}$ and ensure the conductivity of the electric field inside the capillary. The separation is achieved strictly due to differences in electrophoretic mobilities between the separated analytes. Because in similar conditions, electrophoretic mobilities are influenced by the charge and hydrodynamic radius, CZE generates a separation radically different to the ones conventionally accessible using LC. Early in the introduction of mAb conjugates, CZE was evaluated as an analytical technique for their characterization. Thus, Liu and co-workers developed a CZE-UV/LIF method that could be successfully used for the analysis of an ADC composed of DOX linked through cysteine residues via a hydrazone linker. Following tryptic digestion, the peptide mapping was performed and compared to the equivalent experiment, corresponding to the 'naked' mAb. Also, the fluorescence detection was used to specifically monitor DOX conjugated peptides. The results showed the possibility to perform robust peptide mapping using the developed CZE-UV/LIF method. The sensitivity provided by the fluorescence detection allowed the detection of the different conjugated peptides for quantities as low as 30 amol. In addition, free DOX moieties could be detected showing the versatility of this analytical methodology. Finally, the CZE-UV/LIF was used consequently to offline RPLC prefractionation which emphasized the possibility to apply this type of instrumental approach for more complex ADC samples [94].

CZE can also be implemented for the separation of intact ADCs. Kobuta et al. have described in a recent article, the optimization and statistical validation of a CZE-UV method for the analysis of ADC charge variants. The results reported showed the method could be applied for different types of ADCs with comparable performance regarding charge variant separation. Note, the separation obtained was not generated from dissimilar values of conjugated drugs between the variants but rather on the occurrence of PTMs inducing charge variation like glutamic acid cyclization or deamidation. Therefore, this method revealed to be a relevant tool to focus simply on this type of PTMs, regardless of the heterogeneity induced by the yield of the conjugation reaction. The method could be implemented for the analysis of stability samples demonstrating the gradual degradation of ADC samples submitted to forced temperature stress. The validation of the method allowed in parallel to emphasize the excellent 
linearity of the signal over the studied concentration range as well as satisfying LOD/LOQ and the robustness of the CZE-UV method could be illustrated for different ADCs. Therefore, the CZE-UV method demonstrated to be fully compatible with ADC quality assessment and could represent a satisfying alternative to investigate the introduction of several types of PTMs on the peptide backbone of ADCs [95]. To further improve the resolution of the separation, Henley and coworkers have designed a CE instrumentation capable of delivering ultra-high voltage (UHVCE). This instrument can maintain a stable electric field twice higher compared to conventional CE instrumentation. Using a similar zwitterionic background electrolyte (BGE), UHVCE-UV analysis was performed for a model ADC. The results showed a significant improvement in the separation of ADC charge variants regarding peak width and resolution, enabling in several cases to distinguish additional species [96].

Consequently, to the introduction of ADCs, an analytical method based on CZE separation could be developed to characterize the primary structure of this type of therapeutic proteins. At the peptide level, CZE allows performing robust peptide mapping which proved to be orthogonal to RPLC, for instance, to assess the conjugation reaction. Concerning intact ADC analysis, CZE allows investigating the heterogeneity originating from charge variants. Because the type of conjugated drugs is constantly diversifying, CZE could potentially be further relevant to study the heterogeneity inherent to the conjugation process. Especially, in the context of drugs exhibiting charges in solution which could lead to their separation under the influence of the electric field. 


\section{State-of-the-art mass spectrometry approaches}

The native MS approach, formerly applied for the characterization of intact noncovalent protein/protein [97] or protein/ligand assemblies [98,99], has considerably evolved since its development in the early 90s. Native MS has been shown to be a forefront technique for rapid, sensitive and valuable analytical characterization of mAbs [100-109] and ADCs [58,109-112] at the intact protein level [113]. For ADCs, native MS provides information on various CQAs, including glycoprofiles, DLD, DAR ${ }_{a v}$, amount of unconjugated drug (D0), aggregation species. When coupled to ion mobility (IM), IM-MS afford additional conformational characterization of all MS detected species [114,115].

\subsection{Native MS}

Native MS has been first shown to be highly informative for Cys-ADC product, constituted of a mixture of covalent and non-covalent D0 to D8 populations because of the chemical bioconjugation process $[111,116,117]$. The benefits of native MS have also been reported on covalent Lys-ADC, taking into account the narrower charge envelope distributions shifted towards greater mass-to-charge $(\mathrm{m} / \mathrm{z})$ values in native conditions compared to MS data under denaturing conditions (bringing enhanced separation between charge states) to distinguish overlapping drug-load species [58]. Altogether, native MS can now be routinely used for screening of bioconjugation process, including the chemical conditions used for reaction on both Cys- and Lys- ADC compounds [118,119]. In addition to being rapid, accurate and sensitive, native MS is also versatile, as it can be applied to a vast variety of ADC formats including more homogeneous $3 G$ site-specific [120] and rebridged ADCs [119]. Originally hampered by the mandatory buffer exchange sample preparation step necessary prior to native MS analysis, the methodology has considerably evolved and benefits now from fully automated workflows. SEC can indeed be used as a fast desalting tool (providing separation of nonvolatile, non MS-compatible salts from the ADC sample), and coupled in-line to native MS, to automatize sample preparation and increase throughput. High throughput can be reached by using small SEC cartridges $(3-5 \mathrm{~cm})$ with reduced particle sizes $(<3 \mu \mathrm{m})$ affording SEC-native MS runs within less than five minutes [121]. Online hyphenation of SEC to native MS not only ensures automated sample handling and data acquisition but also opens the way for including statistical analysis coming from replicate measurements. In parallel, progresses have been made in bioinformatics pipelines provided by manufacturers, most of them now including user-friendly modules for accurate and automated mass and average DAR calculation (mostly from deconvoluted MS data), that can handle heterogeneous/complex MS data interpretation or replicate comparisons [122]. Mass accuracies below $50 \mathrm{ppm}$ and improved dynamic ranges are now routinely obtained on high-resolution native MS platforms (FTICR [123], orbitrap [58,111,120] or Q-TOF [121]) that allow unambiguous determination of 
some of the main CQA of ADCs (i.e., relative amount of D0, DAR $\mathrm{av}_{\mathrm{av}}, \mathrm{DLD}$ ) within one unique 5 min LC-MS run in non-denaturing conditions. Thanks to all these recent technological and bioinformatics breakthroughs, high-resolution native MS has entered most world leading biopharma companies and is now routinely used in R\&D labs for straightforward ADC analytical characterization. To highlight the versatility of the SEC-native MS approach, Figure 2 presents online SEC-native MS analysis of different types/generations of ADCs, including Cys- and Lys$A D C$ in addition to new generation site-specific and rebridged ADCs. In all cases, from rather homogeneous to highly heterogeneous ADCs, DLD, average DAR, and unconjugated D0 amounts could be deduced and agreed with chromatographic methods. This combination of SEC to native MS can potentially be extended to forced degraded studies. Indeed, separation capabilities of SEC columns can be exploited to provide chromatographic separation of LMWS and HMWS before online native MS identification. Ehkirch et al. have recently published a proof of concept of SEC-native MS benefits for forced degraded studies of mAbs [121].

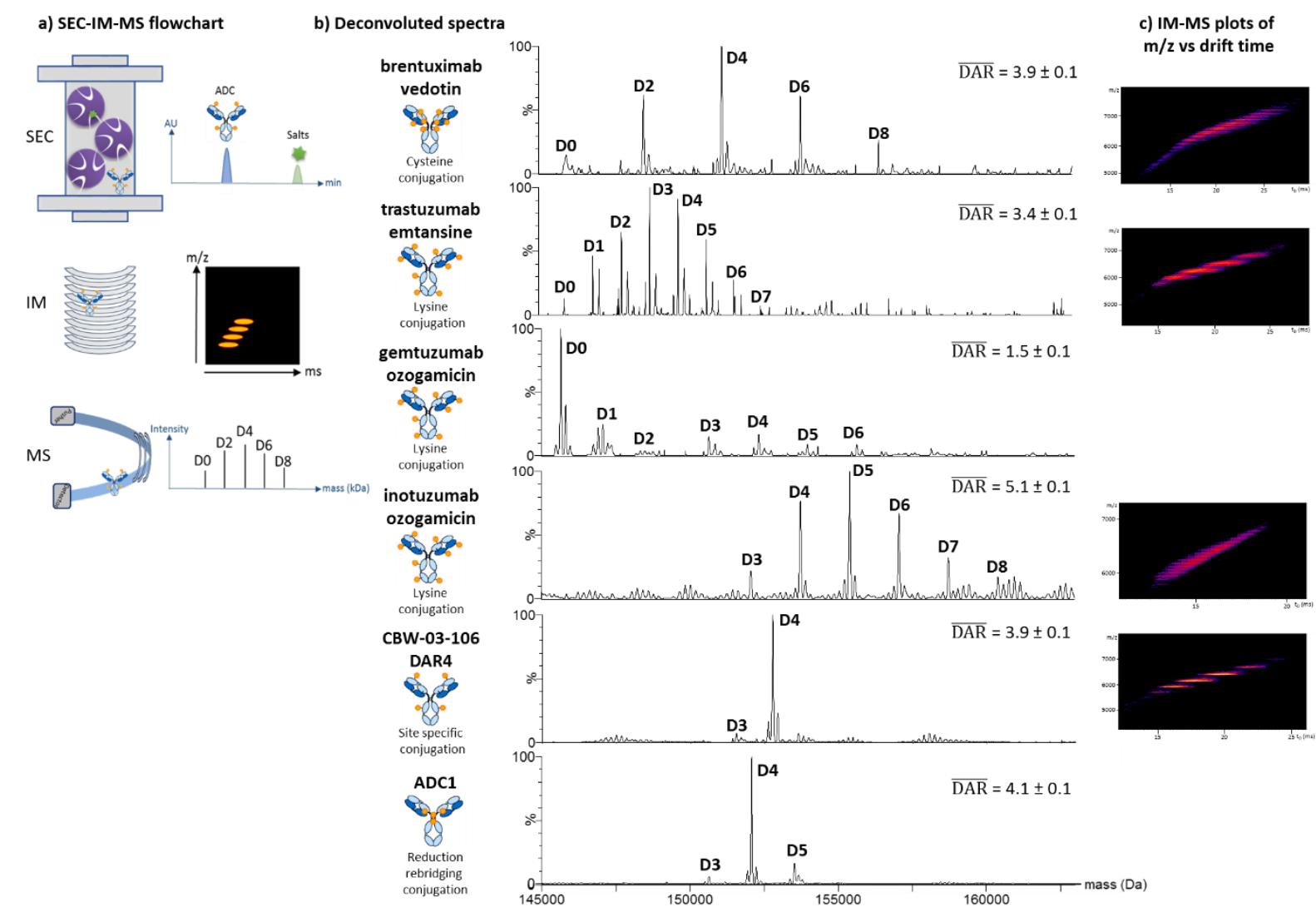

Figure 2. Online SEC-native IM-MS analyses of ADCs (Cysteine, Lysine, Site specific and reduction-rebridging conjugations). a) SEC-IM-MS flowchart. b) Deconvoluted spectra and c) IM-MS plots were presented for each individual ADCs. 


\subsection{Hyphenation of non denaturing LC to native MS}

The success of online SEC to native MS has stimulated interest for more generally coupling non-denaturing chromatographic (like HIC or IEX for hydrophobic and charge variant analysis, respectively) or electrophoretic (like capillary zone electrophoresis CZE [44,124-126]) techniques classically used in QC labs to the power of native MS. Those non-denaturing LC methods often require high amount of nonvolatile salts within the mobile phase for optimal chromatographic performance $[46,127]$, which are not directly compatible with native MS. A first way to circumvent this limitation is to add a second dimension of chromatographic separation, aiming most often at including a reversed phase $\mathrm{C} 18$ column for sample desalting [128-131] and further classical denaturing MS analysis (see sections 5.1 .5 and 5.1 .6 on multidimensional LC). As an alternative to 2D-LC setups, several groups suggested performing non-denaturing LC (SEC, HIC or IEX) using ammonium acetate as mobile phase, to use 1D LC-native MS setups.

For charge variant analysis, two approaches have been described in the literature to afford online IEX-native MS. A challenge for $\mathrm{pH}$ gradient IEX is to find a balance between low salt concentrations for optimal MS sensitivity while maintaining control of the $\mathrm{pH}$ to facilitate reproducible chromatography and have suitable peak shapes [132]. When coupled with native MS, separation using the $\mathrm{pH}$ gradient approach was found to outperform salt gradient separation on a strong cation exchange phase. Limited reports of the direct hyphenation of IEX to MS exist in the literature, the main bottleneck for performing IEX in ammonium acetate being the high amount of ammonium acetate required (> $200 \mathrm{mM}$ ), which negatively affects native MS sensitivity and resolution [132]. Online hyphenation of IEX to native MS using a single $\mathrm{pH}$ unit gradient change on a weak cation exchanger using ammonium hydroxide eluents containing $20 \%$ methanol has been described first for intact mAb analysis [133] and more recently for middle level characterization [134], which are not stricto senso "nondenaturing" conditions as $20 \%$ methanol can affect mAb conformation. Conversely, Füssl et al. have reported the use of low ionic strength $\mathrm{pH}$ gradient elution of $\mathrm{mAbs}$ from a strong cation exchanger coupled to high-resolution native Orbitrap mass spectrometer for high-resolution, high-definition characterization of mAb charge variants $[135,136]$. This time elution buffer was consisting of $25 \mathrm{mM}$ ammonium bicarbonate and $30 \mathrm{mM}$ acetic acid as buffer $\mathrm{A}$ ( $\mathrm{pH}$ 5.3) and $10 \mathrm{mM}$ ammonium hydroxide in $2 \mathrm{mM}$ acetic acid as buffer $\mathrm{B}(\mathrm{pH} 10.18)$, which again are not $100 \%$ non-denaturing [135]. Bailey et al. described a similar workflow that simultaneously accomplishes native intact mass analysis on a high-resolution native MS orbitrap platform and charge variants analysis using weak cation exchange performed in robust mobile phase 
buffers and is based on $\mathrm{pH}$ gradient separation using extremely high purity MS compatible buffers at low ionic strength. For this purpose, a pH-elution IEX method with low ionic strength and a constant background of volatile salt, which served to minimize gradient-related changes in ESI sensitivity and to maintain equivalent desolvation conditions throughout the entire course of separations was developed ( $50 \mathrm{mM}$ ammonium acetate without $\mathrm{pH}$ adjustment as mobile phase $\mathrm{A}$ and with $\mathrm{pH}$ adjustment using ammonium hydroxide in mobile phase $\mathrm{B}$ ) [137]. Even if it has not yeet been reported, the IEX-MS approaches described in this section could potentially be applied to lys-ADC for the characterization of CQAs such as the DLDs, average DAR and amount of unconjugated mAb.

Direct coupling of hydrophobic Interaction chromatography (HIC) and native MS is also attracting, especially for Cys-ADC characterization where HIC is a quality control method for establishing DLDs, average DAR and amount of unconjugated mAb. Again, HIC elution can be performed at high concentrations of ammonium acetate (often also in the presence of organic modifiers, that are not stricto senso native) and directly coupled to native MS. For instance, Chen et al. described the direct coupling of HIC to native MS but with a mobile phase containing denaturing/organic solvent (eg linear gradient from 99\% MPA (1 M ammonium acetate) to $99 \%$ MPB (20 mM ammonium acetate in 50\% ACN) which might not be appropriate for cysteine ADC analysis [44,138].

As ammonium acetate does not always afford best chromatographic performance compared to classical salt-containing buffers, alternative methods involving innovative multidimensional LC setups coupled to native MS were developed and are detailed in sections 5.1.5. and 5.1.6.

\subsection{Native IM-MS}

Over the past decade, IM-MS has emerged as an orthogonal analytical technique to bring conformational characterization of protein complexes [139], including mAbs [102,140,141] and ADCs $[58,111,120]$. IM separates ions based on their size to charge ratio and reports ion size in terms of a rotationally-averaged collision cross section (CCS). IM-MS is a promising approach for biotherapeutic characterization as it combines advantages of conformation-based separation of IM to the accuracy of MS measurements.

Since IM provides a global view of conformational differences, it is a good choice for preliminary investigations of mAb-based product conformations, where the shape and size of the molecule are of interest. For ADC characterization, IM can either be used qualitatively mostly to address heterogeneity issues, but also in a quantitative way, to determine CCS values or to directly evaluate the relative amounts of the different species present in solution from IM data, providing reliable CQAs information (DLD, DAR ${ }_{\mathrm{av}}$, unconjugated D0). 
IM-MS usually begins with CCS measurements of therapeutic proteins after carefully controlling and adapting calibration procedures depending on the IM instrumentation that is used. Those experimentally IM-derived CCSs can be further compared to calculated theoretical values coming from an equation linking CCS and MW [142], deduced from 3D structures or after MD simulations [143]. CCS measurements are often the starting point for $\mathrm{mAb}$ and $\mathrm{ADC}$ analysis, but their "effective" usefulness for therapeutic protein characterization can be questioned as few or no 3D structures are reported in the Protein Data Bank (PDB, https://www.rcsb.org/pdb) for mAbs and ADCs, respectively. However, CCSs reported by different independent groups were consistent, which demonstrates the robustness of IM-MS measurements. So, accumulation of IM-derived CCS values will serve for the constitution of a broad library of reference values, waiting for 3D structures for more in-depth/extensive data interpretation. Of note, the experimentally IM-estimated CCS values were shown to be significantly lower than the CCS determined either from crystal structures or from MD simulation for ADCs $[111,120]$ and for mAbs $[58,141,144]$. This is explained by the flexibility of the hinge region that collapses in the gas phase, such that the measured CCS of the mAb is substantially less than both the predicted CCS from its crystal structure and the in vacuum MD simulation [141]. As expected from the mass effect related to drug conjugation, CCS values of ADCs are usually slightly but significantly higher than those of the corresponding unconjugated mAb $[58,111,120]$. However, drug loads often exhibited similar intact CCS values, which do not allow distinction between a mass effect or a real conformational change induced upon drug conjugation.

IM-MS can be used in an easier and more qualitative way for ADC analysis to provide a rapid and direct snapshot/picture of the conformational heterogeneity/homogeneity resulting from drug conjugation (Figure 2). By comparing $2 \mathrm{D}$ plots of $\mathrm{m} / \mathrm{z}$ as a function of $\mathrm{IM}$ drift times of the $A D C$ versus its former unconjugated $m A b$, clear evidence is provided on the level of heterogeneity/homogeneity of the studied samples, as shown in Figure 2. Debaene et al. have first reported the difference in 2D IM-MS plots of BV compared to the naked antibody building block (brentuximab) [111]. Similar observations were published by Marcoux et al. on Lys-ADC, by comparing 2D plots of T-DM1 and trastuzumab. More recently, Botzanowski et al. reported the high homogeneity of a site-specific $A D C$, like its parent unconjugated mAb, but very different when compared to Lys- or Cys-ADCs [120]. Quantitative data can also be deduced from IM-MS measurements after extraction and integration of arrival time distributions (ATDs) of each individual drug load species, leading to accurate DLD and DAR $\mathrm{av}_{\mathrm{av}}$ assessment. For instance, $\mathrm{DAR}_{\mathrm{av}}$ determined from IM-MS of $3.7 \pm 0.1$ (versus 4.0 expected) [111], $3.4 \pm 0.2$ (versus 3.5 expected) [58] and $3.9 \pm 0.1$ were obtained by IM-MS for BV, T-DM1, and a sitespecific DAR4 ADC, respectively. 
Collision Induced Unfolding (CIU) experiments have proven to be an interesting IM-based alternative to circumvent current limitations in IM cell resolution for therapeutic proteins analysis [113]. CIU is an IM-based gas phase method that aims at collisional heating of ions through the application of an accelerating electric field that results in higher energy collisions with background gas. Ions undergo unfolding without dissociation (no covalent bound disruption). The extent of unfolding is monitored by IM-MS, protein unfolding being measured by corresponding IMdrift time/CCS changes. While IM-MS is often compared to gas-phase electrophoresis, CIU experiments can be seen as a gas-phase analog of differential scanning calorimetry. The comparison of CIU fingerprints (IM drift times as a function of accelerating voltages) from two different samples/conditions can serve to highlight subtly different conformers and to probe differences in their gas-phase stabilities. CIU is a valuable approach to tackle compound with very close conformations, which is the case for the slight conformational changes induced upon drug conjugation for ADCs. While several studies now report CIU experiments to distinguish between mAb isotypes [140,145,146] or for mAb comparability studies [147,148], to the best of our knowledge, only one paper reports on CIU experiments performed on a site-specific ADC. In this study, Botzanowski et al. reported CIU experiment performed on unconjugated $\mathrm{mAb}$ and its corresponding site-specific DAR4 ADC [120]. Different CIU unfolding patterns were obtained for the unconjugated $\mathrm{mAb}$ (three main CIU features) compared to the site-specific DAR4 ADC (only two main CIU states), revealing increased ADC stability toward unfolding as compared with the unconjugated mAb.

Conversely to native MS, and even though automatized IM-MS data acquisition is almost possible and straightforward in SEC-native IM-MS workflows [121], IM-MS is still not adopted in the biopharmaceutical environment for routine conformational characterization of biotherapeutic proteins. Several reasons can account for that. As already explained and despite alternative IM-based experiments like CIU, current IM instruments lack in resolution to tackle major issues related to rapid differentiation of subtly-different ADC isoforms. In the case of ADCs, a challenge expected to be solved by IM-MS is the analytical characterization of positional isomers (ADCs bearing the same number of drugs but at different amino acid positions) in both cysteine and lysine conjugates. Positional isomers are expected to have the same molecular weights but slightly different conformations that might be separated by IM. Such positional isomers have been reported for BV by chromatographic techniques. Unfortunately, current IM cells lack in resolution to separate positional isomers at the intact (150-170 kDa) top level. As no 3D structure of an ADC is available in the PDB, Marcoux et al. used a molecular model of the D4 positional isomers of T-DM1 to see how challenging it would be to separate DAR4 isoforms in an IM cell [58]. Subsequent simulations of the ATDs of the different $D 1$ isoforms indicate that an IM resolution of $\sim 330$ would be necessary to separate 
them in an IM cell, indicating that further instrumental developments are required before the quantitative characterization of positional isoforms by native IM-MS becomes a possibility.

A second explanation for the limited use of IM-MS and CIU for routine use in biopharmaceutical companies is their lack of true high-throughput as compared to native MS. For automated sample injection, native IM-MS has directly benefited from progresses in native MS. Online hyphenation of SEC to native IM-MS has been reported by Ehkirch et al. for mAb and ADC global conformational characterization and in the context of forced degraded studies [121]. This is not the case for CIU experiments. The bottleneck for the moment rather sticks on the sample introduction which is necessarily manual (syringe or microfluidic injection). As highresolution CIU fingerprint data acquisition requires 30-60s acquisitions every $2-5 \mathrm{~V}$, recording of a CIU fingerprint is currently limited by the acquisition time which is thus inherently not compatible with online SEC coupling. In terms of data acquisition, both IM-MS and CIU can be fully automatized. Another bottleneck of the IM-MS approach is automated data interpretation (CCS calculations, 2D IM-MS plots comparisons, etc.) which is still mostly manual and requires strong expertise to avoid misinterpretations. For CIU experiments, the situation is slightly different as the CIU data acquisition process can be fully automatized and included in open source software $[149,150]$ to generate in an easy, rapid and user-friendly manner CIU fingerprints. Those bioinformatic tools also include a statistical comparison of the CIU fingerprints. Finally, IM-MS based experiments still require proper calibration and strong expertise in data acquisition and interpretation which may hamper its routine use in biopharmaceutical R\&D laboratories.

\subsection{Top- and middle-down analysis of ADCs}

One major issue which is not addressed with native MS approaches is the precise location of the drug on the mAb scaffold, as the pharmacokinetics and the stability of the ADCs can be closely related to its bioconjugation site. Primary sequence validation along with localization of drug conjugation is usually performed by classical bottom-up approaches [151]. However, separation and identification of peptides containing the payload in LC-MS/MS analysis are very challenging mainly owing to the hydrophobicity associated with the drug-conjugated peptides [33]. Furthermore prior to bottom-up mass spectrometry, proteins are subjected to long enzymatic digestion that can impact the quality of the final product (increase of oxidation, deamidation, etc. [152]). Improvements on MS resolution and fragmentation techniques have been critical for the implementation of complementary strategies, so-called top- (without any prior sample digestion, TD) and middle-down (formation of 25-30 kDa LC, F(ab)'2 and Fc subunits, MD) analysis to characterize the primary structure of mAbs and related products. Electron-based activation techniques such as ETD and ECD, have been used to induce the fragmentation of intact mAbs due to their higher fragmentation yield compare to slow-heating 
activation techniques (CID) [153] and for the suitability of these techniques to characterize labile post-translational modifications. Despite the remarkable efforts performed on the fragmentation of intact mAbs, less than $40 \%$ of the overall sequence could be characterized [154-159] after merging the results from different experimental conditions, highlighting that further improvements are still needed to allow the implementation of TD MS as a routine method for mAbs and ADCs sequencing. To circumvent the shortcomings associated with TD MS, many groups have envisaged the characterization of unconjugated mAbs after performing IgG protases digestion steps leading to the formation of subunits $[156,159,160]$. IdeS (FabRICATOR) is a cysteine protease that digests antibodies at a specific site below the hinge, generating a homogenous pool of $F\left(a b^{\prime}\right) 2$ and $F c / 2$ fragment and after reduction $L C, F d$, and Fc/2 subunits [156,159,160]. Kgp (GingisKHAN) is a cysteine protease that digests human $\operatorname{lgG1}$ at a specific site above the hinge, generating intact Fab and Fc subunits. The significant sequence coverage achieved using MD MS is not only due to the reduction of the size of the protein but also to the implementation of newly fragmentation techniques such as ultraviolet photo-dissociation (UVPD). Cotham et al. [160] performed MD MS analysis on trastuzumab using ETD, and $193 \mathrm{~nm}$ UVPD. After one LC-MS/MS UVPD run, almost 60\% of the sequence coverage for the three subunits were attained, and after combination with one ETD run, the overall sequence coverage rose to $80 \%$ for $\mathrm{Fc} / 2$, and LC subunits. A similar study was performed by Fornelli et al. [159] either on IdeS-digested or Kgp-digested rituximab. In this case, a commercial orbitrap mass spectrometer equipped with a $213 \mathrm{~nm}$ UVPD laser was used providing very similar sequence coverage compared to those obtained by Cotham et al. and thus, democratizing the use of UVPD for TD and MD MS workflows. While unprecedented outcomes in terms of sequence coverage and time analysis have been obtained in MD MS analysis of mAbs, ADC's remain unexplored by this approach. Hernandez et al. [161] studied the conjugation site of a cysteine conjugated site-specific ADC after IdeS digestion and reduction (MD MS). The MS data associated with the ADC subunits allowed to localize the payload on the Fc, and Fd, while the LC remained unconjugated. However, the conjugation characterization was not only restricted to the subunit level and fragmentation of the Fc, and Fd were performed using three fragmentation techniques (HCD, ETD, and 213 UVPD) to provide the specific position of the conjugation. After 10-min LC-MS/MS run, the conjugation site on both subunits was unambiguously assigned mainly due to the fragmentation efficiency provided by the 213 UV light. Overall, the previous studies were performed under denaturing MS conditions. However, native MS can also be implemented on TD and MD MS approaches. Dyachenko et al. [162] performed tandem MS analysis on a cysteine-linked ADC (BV) under native conditions. Selective fragmentation of high $\mathrm{m} / \mathrm{z}$ molecular ions was carried out in an Orbitrap Exactive EMR with a modified quadrupole working at $284 \mathrm{kHz}$ allowing the isolation and efficient transmission of ions until $20000 \mathrm{~m} / \mathrm{z}$. The positional isomers heterogeneity could 
be assessed after analysis of MS/MS data of specific DAR species of BV. Nevertheless, the success of this workflow was limited to the differentiation between Fab- and hinge-conjugated cysteines without providing further information about the specific conjugation site.

\subsection{Covalent labeling techniques for ADC higher order structure characterization}

Several techniques have been conducted to provide more insights about higher order structures (HOS) of mAbs and ADCs, among which hydrogen-deuterium exchange followed by MS analysis (HDX-MS) is particularly used [163]. Differences in isotope exchange kinetics of amide hydrogens in mAbs have been successfully analyzed to assess the structural impact of post-translational modifications (PTMs) [164,165] while other groups have used this technique to characterize the mAb/Ag interaction (epitope mapping) [166-168]. HOS of mAbs might also be affected by the addition of cytotoxic molecules that could potentially give rise to some undesired effects in terms of safety and efficiency. For instance, HDX-MS was performed to gain further understanding of the impact of drug-linker conjugation to both inter-chain cysteine residues [169] and site-specific engineered cysteine conjugated mAbs [170]. The similar HDX kinetics in both cases led to the conclusions that the conjugation process does not induce large-scale major conformational changes on the mAb backbone, however, local regions where found to be more solvent exposed because of the disruption of the disulfide bonds derived from the conjugation process. The same technique was also used to study the effect of the drug conjugation on Adnectin (a protein scaffold around 15 times smaller than mAbs) [171]. Combination of intact protein HDX, bottom-up HDX, and top-down HDX results did not reveal any significant conformational changes.

Covalent labeling is also considered an alternative approach to HDX that can afford protein structural information related to structure dynamics and solvent accessibility by targeting chemical modifications on the side chain of the amino acids. In contrast to HDX, covalent labeling generates irreversible modifications on protein surface thus making post-labeling sample preparation more flexible. Several covalent labeling techniques have been developed over the years. However, the implementation of these techniques usually implies dedicated equipments and thorough expertise for data analysis. Carboxyl group footprinting (CGF), which is one of the most straightforward covalent labeling technique, was performed to provide conformational insights into mAbs and ADCs [172]. In this case, a glycine ethyl ester tag is added to the carboxyl group of the side chain of the solvent-accessible amino acids. The similar rate constant $(\mathrm{RC})$ of the $\mathrm{mAb}$ and the corresponding Cys-ADC pinpointed the high degree of similarity between both systems. However other orthogonal techniques such non-specific labeling strategies are often required to complement the limited sequence coverage afforded 
by CGF as only Asp and Glu amino acids can be targeted (around $10 \%$ of the overall mAb sequence coverage).

\section{Innovative hyphenated methods}

\subsection{Multidimensional LC coupled with MS}

Analysis of $A D C$ is extremely complex and requires a combination of modern analytical methods and state-of-the-art systems, offering maximal resolving power. In this context, 2DLC is an interesting approach, since it offers high selectivity and resolving power thanks to the use of complementary separation modes. In addition, because RPLC is often one of the selected chromatographic modes, it is preferably used in the second dimension given its compatibility with MS.

There are several ways to implement 2D-LC, which can be ranked in the order of increasing complexity: off-line 2D-LC involving time-consuming fraction collection (out of the scope of the present review article); on-line heart cutting or multiple heart cutting 2D-LC (LC-LC), when only one or few parts of the $1 \mathrm{D}$ chromatogram are sent to the second dimension; and on-line comprehensive 2D-LC ( $L C x L C)$, which is considered today as the most powerful and informative 2D-LC mode [173].

\subsubsection{Heart-cutting and multiple heart-cutting SEC-RPLC-UV/MS for determining free drugs}

To successfully analyze these small molecules cytotoxic drugs without adding a timeconsuming sample pretreatment procedure, 2D-LC can be performed, resulting in an automated workflow and high sample throughput.

Using SEC in the first dimension enables the separation of protein species and small molecules into two very distinct fractions, based on their size. Then, the fraction eluted later in SEC, and corresponding to small molecules can be transferred to the second RPLC dimension using a switching valve, through a heart-cutting approach. The different small molecule species (free drug, linker, linker-drug, process-related impurities and degradation products) can next be separated on the RPLC column. Such an online SEC-RPLC-UV/MS method was reported for the assay of unconjugated free drug and related impurities in ADC product, and for stability indicating assay $[174,175]$. An isocratic SEC elution with the addition of $20 \%$ acetonitrile in the mobile phase was used to elute the free payloads and related species together. Then, MS was used to identify the eluted peaks, while UV was used to properly quantify the small molecule species. This approach was found to be 10-times more sensitive in comparison to the same RPLC method performed after off-line protein precipitation. However, one of the constraints of 
this approach is that it uses an organic solvent in SEC and may therefore denaturate proteins and hamper the determination of high molecular weight species (HMWS).

As an alternative, a multiple heart-cutting SEC-RPLC-UV method was developed for the simultaneous separation of HMWS from the monomeric ADC and for the separation of free payloads from the free linkers and another type of small molecules impurities [49]. For this purpose, the protein species (i.e., HMWS, monomeric ADC, and LMWS) were eluted with less than $5 \%$ acetonitrile. Then, the free payload related species were eluted from the SEC column and separated with an acetonitrile gradient (containing up to $20 \%$ acetonitrile). Where this method was applied to site-specific ADCs, two important CQAs of ADCs can be determined, namely free payloads and HMWS, within only 10 minutes. By using a suitable switching valve, different fractions can be collected, corresponding to the small molecules elution region of the SEC chromatogram. This is illustrated in [49], where numerous small molecular weight species were chromatographically quantified with UV detection, with also the possible identification by MS detection (only volatile salts were used in the RPLC dimension). In the end, the "multiple heart cutting" method significantly increases the peak capacity compared to previously published single heart cutting SEC-RPLC methods.

\subsubsection{Multiple heart-cutting CEX-RPLC-MS for Lys-ADC}

Similarly, to what has been developed for mAbs [129,130], a multiple heart-cutting strategy involving CEX and RPLC in the first and second dimensions was developed for the characterization of Lys-ADC (T-DM1), both at the intact level analysis and after digestion with IdeZ $\left(\mathrm{F}(\mathrm{ab})_{2}\right.$ and $\mathrm{Fc} / 2$ fragments of 100 and $25 \mathrm{kDa}$, respectively). As previously discussed, the individual performance of CEX and RPLC was very poor when analyzing Lys-ADC, due to the complexity and heterogeneity associated with the conjugation (one single broad peak was observed, as shown in Figure 1). To further improve the resolving power of the analytical technique, these two separation modes were combined in a multiple heart-cutting setup.

On the total, seven fractions of the CEX chromatogram of intact T-DM1 were collected and sent to the RPLC dimension. When surveying the RPLC chromatograms of the second dimension, the RPLC retention of the first eluted peaks in CEX was higher, which is logical since more cytotoxic drugs are conjugated to lysine, so hydrophobicity increases, while positive charge decreases. Thanks to the MS information that can be obtained from the second dimension, valuable information could be achieved on the drug load and its distribution on the mAb backbone. It is important to notice that various DAR species can already be slightly separated in CEX (no baseline resolution). In addition, some isomeric species with the drug conjugated at different lysine residues can also be identified. 
When analyzing IdeZtreated T-DM1, in CEX-RPLC-MS, a drug load of 0 to 5 was revealed on the $F(a b)_{2}$ fragments, and the presence of linker without drug was clearly highlighted (this information was not available at the intact level analysis, due to the size and heterogeneity originating from glycosylation).

\subsubsection{Comprehensive RPLC x RPLC-MS for Lys-ADC and Cys-ADC}

A comprehensive RPLC x RPLC-MS-based peptide mapping has been successfully developed for the analytical characterization of mAb samples [176]. This successful strategy has recently been extended for the analytical characterization of Lys-ADC (T-DM1) and Cys-ADC (BV). To achieve highly orthogonal separations of peptides, the two RPLC dimensions were performed at basic and acidic $\mathrm{pH}$, respectively. This 2D-LC approach was shown to be particularly attractive to determine small molecule drug conjugation sites on ADC. Indeed, by comparing the peptide map of $A D C$ and corresponding unconjugated $m A b$, differentiating spots were observed in the upper right corner of the 2D-LC map, corresponding to the most hydrophobic conjugated peptides. Obviously, the specific ions originating from the cytotoxic molecule can help to recognize conjugated peptides with MS.

\subsubsection{Comprehensive HIC x RPLC-MS for Cys-ADC}

With the purpose to obtain the fine structural characterization and the DLD of a Cys-ADC, a detailed two-part series articles were proposed by Sarrut et al. [131,177] for the comprehensive HIC x RPLC-MS analysis of BV. In a first instance, the authors carefully optimized the experimental conditions of both LC dimensions with the goal to (i) prevent salt precipitation, (ii) limit the total analysis time, (iii) increase injection volumes (and thus enhancing sensitivity), and (iv) ensure suitable MS hyphenation. To achieve these objectives, first, different combinations of salts were evaluated, and ammonium acetate was used instead of ammonium sulfate in the mobile phase of the first HIC dimension to avoid precipitation with an organic solvent in the second RP dimension. Then, the 1D-gradient conditions in HIC were optimized and total analysis time of 70 min was achieved with a concave gradient, developed through a computer-assisted gradient optimization software. Finally, to increase the injection volumes, samples were prepared with $2.5 \mathrm{M}$ ammonium acetate in the injection solvent to increase their ionic strength prior to the injections and thus to obtain the decrease of their eluent strength. By applying this strategy, it was possible to inject up to $40 \mu \mathrm{L}$ without occurring in chromatographic peak distortion, while achieving enhanced sensitivity. Then, to maximize peak capacity in the second dimension, both high temperature and high flow rate were used for RP separation. However, prior hyphenation with MS, the flow rate was diverted to discard salt contamination and then decreased and redirected to the MS to achieve sufficient sensitivity. Using this optimized HIC x RPLC strategy, the coupling with high-resolution MS 
was performed, and structural information on positional isomers of BV DAR species was obtained at an unprecedented level of details. In fact, once obtained the separation of the native DAR species in 1D HIC dimension, each DAR was additionally analyzed in the 2D RP dimension and thus non-covalent bound $A D C$ sub-units were separated thanks to the denaturing nature of the RP mobile phase and unambiguously assigned by MS. Finally, HIC $x$ RPLC profiles of BV were compared with the profiles of one- and two-month stressed samples. Interestingly, in addition to even DAR, odd DAR species were detected in the stressed samples and their relative abundance assessed. Notably, HIC x RPLC contour plots were found to be a particularly useful representation for assessing the structural differences among stressed and non-stressed samples.

\subsubsection{Comprehensive HIC x SEC-IM/MS for Cys-ADC}

An innovative multidimensional analytical approach has been recently proposed by Ehkirch et al. [178] to achieve the on-line coupling of HIC to MS for the structural characterization of BV (Figure 3). Specifically, comprehensive 2D-LC, consisting of HIC in the first dimension and SEC in the second dimension, was coupled online to IM-MS for performing detailed and streamlined characterization of both native and forced degraded ADC samples. The main goal during the development and the optimization of this $4 \mathrm{D}$ configuration was to maintain the integrity of the sample upon HIC separation and prior native MS analysis, and thus obtaining a full online characterization performed in non-denaturing conditions. Through the optimization of the second dimension, the authors were able to successfully achieve this goal. In fact, SEC was solely used for its size-based separation capabilities and exclusively applied as "fastdesalting" step prior to MS detection.

1D HIC chromatographic conditions were optimized to obtain the best peak resolution, and mobile phases containing non-volatile salts were used (A was $2.5 \mathrm{M}$ ammonium acetate, and $0.1 \mathrm{M}$ phosphate buffer and $\mathrm{B}$ was $0.1 \mathrm{M}$ phosphate buffer). Then, 2D SEC separation was performed on-line in isocratic mode by using volatile mobile phases fully compatible with native MS, consisting of $100 \mathrm{mM}$ ammonium acetate solution. Large species, namely DAR moieties, were eluted prior small species (salts) within a total SEC analysis time of $1.5 \mathrm{~min}$. DARcontaining SEC fraction were then sent to the MS, while the remaining fraction containing the salts was redirected to waste prior flow entry into IM-MS instrument, thanks to the use of an external switching valve. Within a single run, a global IM-MS picture was also provided. The $4 \mathrm{D}$ platform was used to characterize BV in comparison with a one-month stressed sample. An in-house ADC under development was also used to illustrate the benefits of the $4 D$ HICXSEC-IM-MS workflow compared to HIC or native MS used as standalone techniques. Altogether, the results demonstrate for the first time the ability to have a comprehensive 
analytical characterization of a Cys-ADC within a single run, affording: i) simultaneous DLD and quantitative $\mathrm{DAR}_{\mathrm{av}}$ assessment $(\mathrm{HIC})$; ii) unambiguous identification of the number of drug conjugations through accurate intact mass measurement (native MS); and iii) conformational homogeneity assessment of each drug load species (IM). To highlight the relevance of the direct hyphenation of HIC to native MS through HICxSEC-native MS approach, Figure 3a presents the characterization of an in-house Cys-ADC under development. HIC separation allows the differentiation of D4 positional isomers, thanks to the MS-based unambiguous identification and also the identification of minor peak as D3. Finally, it is important to notice that the online HICxSEC-native MS provides an accurate DAR av $_{\text {ov }}$ 4.1.

\subsubsection{Comprehensive SEC x SEC-IM/MS for cysteine conjugated ADC}

Another non-denaturing chromatography hyphenated to native MS could be envisioned, namely SEC for size variants determination, since it is a major CQA. Classical SEC using nonvolatile mobile phases are not directly amenable to native MS. The hyphenation of SEC to native MS, employing volatile mobile phase such as ammonium acetate was described in section 2.1. for direct identification of each SEC peaks. Nevertheless, the use of volatile mobile phase could decrease considerably SEC performance, particularly when analyzing hydrophobic ADC products. Based on the previous observation, a recent multidimensional analytical approach was proposed, combining online two-dimensional SECxSEC (1D as separation step, 2D as fast desalting step) with ion mobility and mass spectrometry in nondenaturing conditions, affording i) optimal SEC performance (under classical non-volatile salt conditions), ii) online native MS identification and iii) IM-MS conformational characterization of all separated size variants. As depicted in Figure 3b, online SECXSEC-native MS analysis of an in-house ADC is presented. HMWS and LMWS are separated with 1D SEC and online detected as dimer, monomer, Fc-Fab and Fab fragments. Finally, a precise relative quantitation of HMWS (2.1\%) and LMWS (0.8\%) could also be established based on the 1D SEC chromatogram. 
a) HICXSEC-native MS

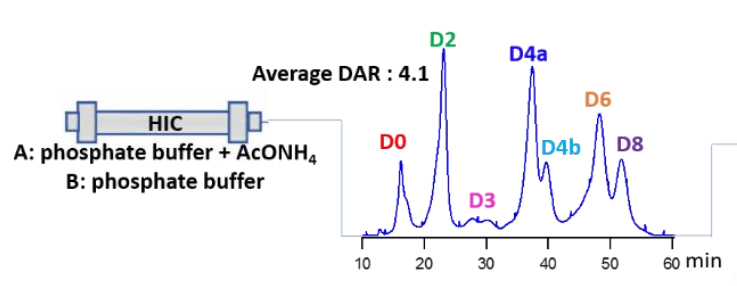

b) SECxSEC-native MS

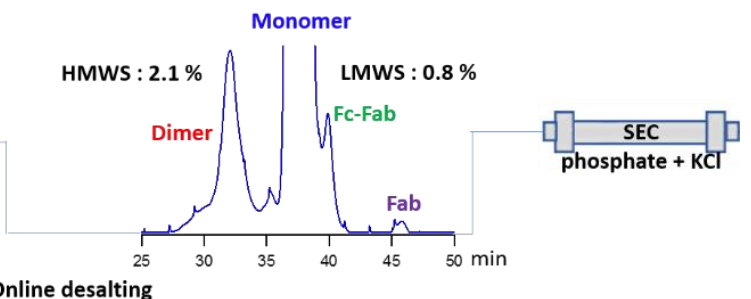

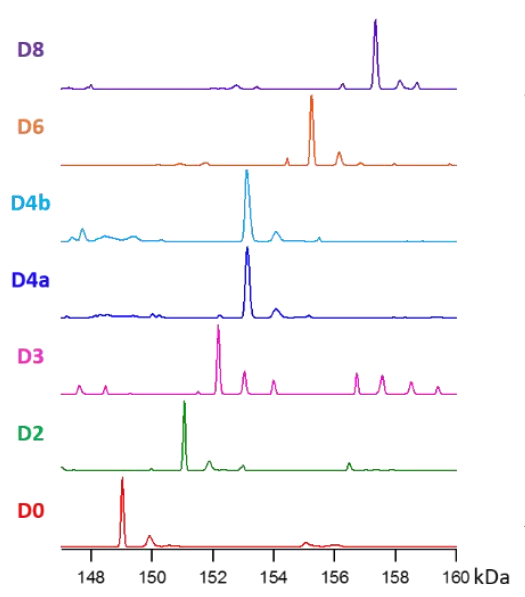

$\mathrm{AcONH}_{4}$ of each fraction

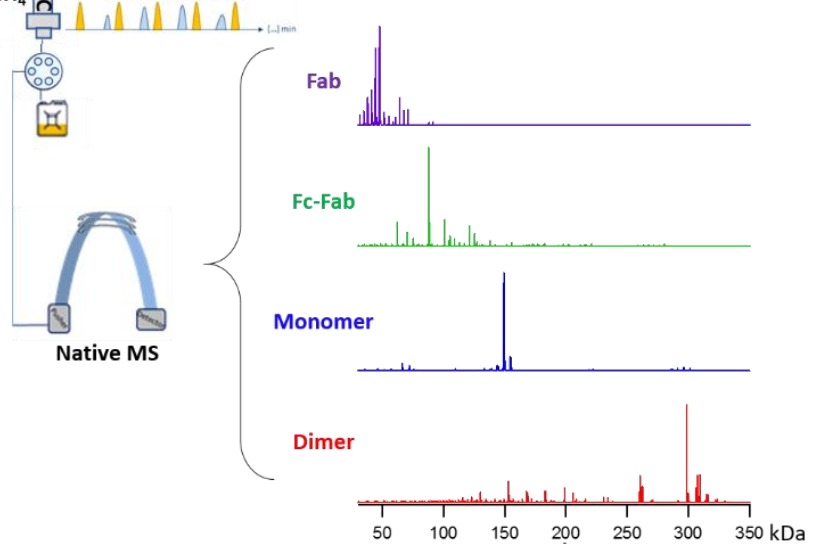

Figure 3. On line coupling of non-denaturing chromatographic methods to native MS. a) Online HICxSEC-native MS of ADC1 with HIC profile and deconvoluted mass spectra of each 1D HIC species. D4 positional isomers are separated by HIC, fast desalted by SEC and identified by mass measurement in native MS. b) Online SECxSECnative MS of ADC2 for charge variant analysis, with SEC profile and deconvoluted mass spectra of each 1D SEC species. The first SEC dimension is performed in phosphate buffer, the second SEC dimension is a fast desalting step while SEC separated HMWS and LMWS are identified by native MS.

\subsection{Combining capillary electrophoresis and mass spectrometry}

In recent years, CE-MS has demonstrated an increased interest, especially for the characterization of biopharmaceutical products including ADCs. The appeal for this technique is partially attributed to the introduction of CE-MS instrumentation, which improved the compatibility between the two techniques. Indeed, contemporary CE-MS instrumentation enables to exploit to the fullest the unique selectivity provided by the electrophoretic separation and achieve the outstanding sensitivity provided by high-end MS instruments $[179,180]$.

Said et al. developed a multi-level analytical methodology using CE-MS for the characterization of the primary structure of ADCs. The approach, applied to the characterization of BV, was based on the combination of intact, middle-up and bottom-up analysis. The results allowed determining the relative abundance of each species exhibiting different conjugated drugs. The relative distribution obtained enabled to deduce the $\mathrm{DAR}_{\mathrm{av}}$ of the sample [181]. In conjunction, the ADC proteolytic digest generated through a specific protocol was characterized using CZEMS/MS. Results allowed in a single experiment the characterization of the amino acid 
sequence with a complete sequence coverage and to the major PTMs including glycosylation (Figure 4a-c). In addition, the same dataset allowed to precisely localize the conjugated drugs on the peptide backbone of brentuximab (Figure 4d) which consequently demonstrated the ability to estimate the yield of conjugation for each site independently as well as identify minor conjugation sites [182]. This CZE-MS/MS method showed to be particularly powerful for the in-depth characterization of ADCs over the different level defining the primary structure of the protein and could be further evaluated for the analysis of ADCs relying of different types of conjugation processes.
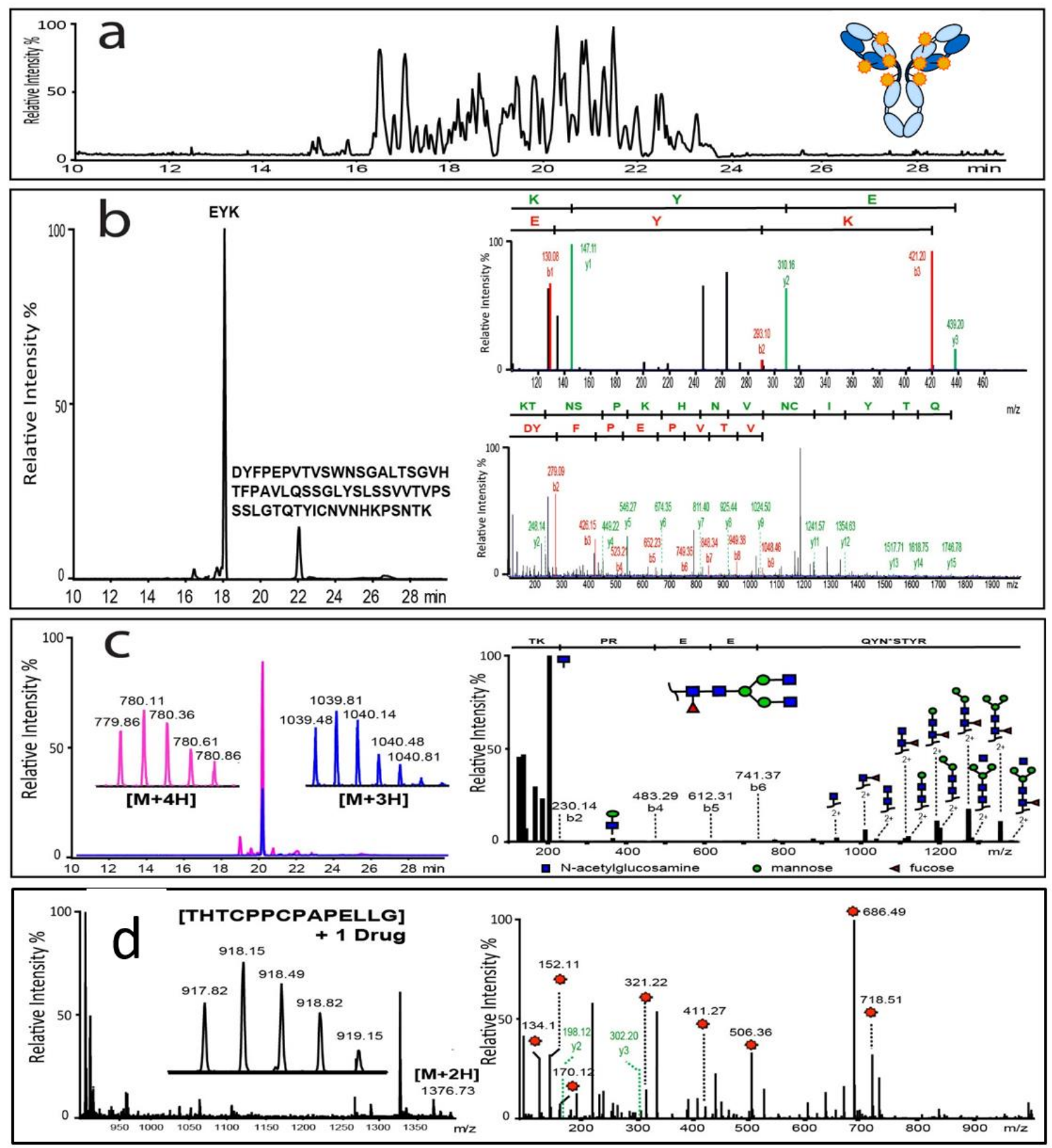

Figure 4. Characterization of the ADC amino acid sequence and its major PTMs including glycosylation performed by CZE-MS/MS. a) Base Peak Electropherogram (BPE) corresponding to the analysis by CESI-MS of brentuximab tryptic digest. b) Extracted Ion Electrophorogram (EIE) corresponding to the $\mathrm{m} / \mathrm{z}$ ratios of [EYK] and 

MS/MS spectra of drug-loaded peptides. [THTCPPCPAPELLG] + 1 payload. Reprinted from [182]. Copyright (2016) Elsevier.

Furthermore, Dada et al. have described an alternative CZE-MS method used for the characterization of the primary structure of an ADC incorporating PBD dimer conjugated by the intermediate of cysteine residues. Following proteolytic digestion, the peptide mixture was characterized by CZE-MS using a BGE containing a minor portion of the organic solvent to enhance the separation selectivity. Achieved results demonstrated complete sequence coverage emphasized by the systematic identification of an extended variety of peptides regarding their chemical nature in concomitance to the highly hydrophobic peptides bearing PBD drug. Also, the most abundant glycopeptides could be identified. The confrontation with RPLC-MS analysis for the same ADC peptide mixture demonstrated the orthogonality between the two instrumental approaches [183].

Due to the electrophoretic mobilization of the analytes, CZE is also adapted to the separation of intact mAbs and ADCs [184-186]. Redman and coworkers developed an integrated CE-MS instrument based on a microfluidic $C E$ device which could be successfully implemented for the intact analysis of a Lys-ADC. The described results showed the separation thanks to CE, of the five main species exhibiting different values of conjugated drugs. Therefore, the CZE-MS analysis enabled the identification of the number of conjugated drugs for each species and the determination of the average DAR value for the studied sample [187].

The research activities regarding the development of CE-MS is particularly vivid and should provide the introduction of more applications adapted to the characterization of ADCs soon. As an example, recent CE-MS applications rely strictly on the implementation of CZE concerning the separation. Innovative instrumentation such as 2D CE-MS opens the opportunity to perform CIEF separation in the first dimension followed by CZE in the second dimension, with online coupling to high-resolution MS [188,189]. This type of instrumentation has the potential to further increase the level of characterization achieved in a single experiment. These perspectives appear particularly adapted to address the additional heterogeneity arising from ADCs compared to conventional mAbs. 


\section{ADC Bioanalytics}

Bioanalytical methods are rapidly being developed to quantitatively monitor the transformation of ADCs in various in vitro or in vivo biological matrices such as serum/ plasma [190] and tumor tissues [191]. Indeed, a crucial property of conjugates is their stability in biofluids as the release over time of cytotoxic drugs into the bloodstream constitutes a considerable health threat [192]. This drug loss also affects the composition of the ADC, potentially altering the amount of drug delivered to the tumor site and posing another substantial safety risk because of its off-target toxicity [193]. To fully describe the pharmacokinetics (PK) of an ADC, several analytes are commonly quantified, including total antibody, conjugate, and payload [194-196]. Among them, the conjugate is the most challenging to measure, because it requires detection of both small and large molecules as one entity. Existing approaches to quantify the conjugated species of ADCs involve a ligand-binding assay (LBAs) for conjugated antibody or hybrid LBA/liquid chromatography/tandem-mass spectrometry (LC/MS/MS) for quantitation of conjugated drug. Now, multilevel LC-MS workflows are more frequently used both for $2 \mathrm{G}$ $[197,198]$ and $3 G$ ADCs $[199,200]$ as well as for protein-drug conjugate (PDC, such as centyrin scaffolds [201,202], based on immune-enrichment [203,204] and high-resolution MS [205]. Metabolites and catabolites of the drug molecule, with or without the linker, may also be detected [206-211]. Pharmacokinetic profiling highlights the impact of the DAR [212] and other perfectible parameters on the biological properties of ADCs, notably the rate of drug loss (from deconjugation and instability) and the clearance of the species with different DARs [213]. Ultimately though, establishing the relationships between pharmacokinetic exposures and the efficacy and toxicity of the drug is the most helpful guide for the optimization and development of ADCs [191,214]. 


\section{Expert commentary}

The multi-level state-of-the-art LC, CE and MS methods currently used to characterize mAbs, are translating to the study of ADCs (HMWS, intact, LMWS, subunits, peptides, SMDs).

The development of high resolution (HRAM) instrumentation, complementary fragmentation methods, and optimized sample preparation and workflows, have allowed MS to grow and to be the leading ADC identification and characterization technique.

Multidimensional LC-MS (from 2 to 4 dimensions) facilitates online studies of large molecules such as mAbs and ADCs, thanks to the extra resolution enabling the direct identification of numerous species and important CQAs [215]. The first dimension can be adapted to the problem at hand via the use of different chromatographic techniques developed for the characterization of mAbs and ADCs (i.e., SEC, HIC, AEX, RP, HILIC), while RPLC or SEC are mostly used in the second dimension to desalt the sample before its introduction into the MS (under denaturing and native conditions, respectively). Native ion mobility and mass spectrometry (IM-MS) may be used in the last dimensions to gain structural insights.

Collision-induced unfolding (CIU) experiments can be successfully used to compare the gasphase conformational stability of parental mAbs vs. ADCs.

The middle-down analysis is now a routine method that can supplement intact mass analysis of $m A b s$ and ADCs and peptide mapping. This method utilizes digestion enzymes with minimal cleavage sites, such IdeS or IdeZ, which split the IgG-based product into subunits ( $25 \mathrm{kDa}$ after reduction) for LC-UV/MS analysis or prior to MS/MS sequencing. HILIC and CEX are used as orthogonal methods to RPLC for ADC subunits analysis.

In addition, top-down MS and sequencing [154,159,216], middle-down MS with high energy collisional-, electron-transfer and ultraviolet photo-dissociation (HCD, ETD and UVPD) fragmentation, are all in constant progresses.

Capillary electrophoresis coupled to MS is now also a well-established method, orthogonal to LC, for peptide mapping at the bottom and middle levels, and progress toward top-level analysis is forthcoming, including native conditions.

\section{$x x x x x x x x x x x x x x x$}

As summarized throughout this review, accumulated reports clearly indicate strong evidence that emerging analytical and structural approaches will successfully contribute toward developing more stable, safer and efficacious ADCs. 
As previously done for mAbs $[36,73,74]$ and reviewed here, current FDA and EMA approved ADCs (BV, T-DM1, IO, GO) may be used as reference materials and benchmarks to evaluate performance metrics for new analytical workflows.

\section{Five -year view}

In the last 5 years, the development of ADCs has benefited from general improvements in the design of therapeutic mAbs and from specific improvements in methods for conjugate synthesis as well from the structural, analytical and bioanalytical methods reviewed here.

ADCs can increase the efficacy and decrease the toxicity of their payloads in comparison with traditional cytotoxic drugs. The targeted delivery of cytotoxic drugs to cancer cells increases the percentage of drug molecules that reach a tumor, thus lowering the minimum effective dose and increasing the maximum tolerated dose. Nevertheless, the therapeutic window remained narrow for most of the current $2 G$ ADCs. This is due to off-target toxicity, which is frequently linked to retro-Michael deconjugation, competition with unconjugated antibody and aggregation or fast clearance of conjugates with DAR of 8 .

3G ADCs are designed to expand the therapeutic window. The toxicities that have been reported for active and discontinued drugs, as well as the optimization of the antibody, the linker, and the conjugation chemistry, are important to drive the rational design and improve the therapeutic index of $3 G-A D C s$.

Moreover, alternative formats to mAbs, such as small protein scaffolds (DARPins, nanobodies, single-chain variable fragments (scFvs), peptide-drug conjugates [217], antibody-dual-drug conjugates (ADDCs), Fabs, Probodies and highly loaded ADCs such as Fleximers (up to DAR 12 to 15)), are now being investigated at early clinical trials. Most of these new formats are more complex than the current $A D C s$ that have reached the market and need a continuous improvement of structural and analytical methods as discussed here.

In addition, new applications for ADCs have been explored outside the field of oncology using non-cytotoxic drugs and will probably increase in the future [218,219]. 


\section{Key issues}

- The inherent complexity of ADCs, which results from the heterogeneity and PTMs, the linker-payload conjugation, combined with changes during manufacturing processes, purification and storage necessitate advanced methodology for their characterization.

- High-resolution native MS provides accurate mass measurements (within $30 \mathrm{ppm}$ ) of intact ADCs and can also yield the drug load distribution (DLD) and average DAR. Native MS is furthermore unique in its ability to simultaneously detect covalent and noncovalent species within a mixture.

- Native IM-MS reveals the drug-loading profile of ADCs, the CCCs of each payload species, highlighting slight conformational differences.

- As an orthogonal method, LC-MS following IdeS digestion of ADCs can be used to measure the drug-load distribution on light chain and Fd fragments, as well as the average DAR for both monomeric and multimeric species. In addition, the Fc fragment can be analyzed in the same run, providing a complete glycoprofile and demonstrating the presence or absence of additional conjugation.

- The linker-payload and glycan modifications of Cys linked ADCs can be simultaneously characterized using a HILIC-MS middle-up analysis, which is orthogonal to RPLC-MS.

- Because the drug molecules are frequently hydrophobic, all enzymatic digestion steps used to prepare the sample for peptide mapping have to be adapted to keep the drugloaded peptides soluble. When the procedures are optimized, unambiguous maps are obtained by LC-MS, while the positional isomers of the cytotoxic drug can be determined by RP-HPLC after digestion with IdeS and reduction.

- Multiple Attributes Method (MAM) based on HRMS data with automated identification and quantitation to monitor CQAs in one single analysis for mAbs may also be used for ADCs.

- All these methods are also useful for comparability [220] and forced degradation studies [221].

- On the bioanalytic side, LC-MS quantitation of larg biotherapeutic proteins such as mAbs and ADCs at the intact level presents many challenges in both LC and MS due to their high molecular weight, large size, structural complexity, and heterogeneity.

- ADCs can be quantitated at the intact level with highly selective sample preparation combined with LC-HRMS analysis. Technological advances in processing software, protein chromatographic columns, ionization techniques, and high-resolution mass spectrometry are still required to successfully quantify large intact proteins at much lower levels. 
- Due to the implementation of an electrophoretic separation, CE has demonstrated to be particularly relevant for the characterization of ADCs. Depending on the separation mode applied, CE can provide, on the intact or/and reduced ADCs level, the analysis of a substantial number of micro-heterogeneities which includes size or charge variants, the number of conjugated drugs, glycoforms and conventional PTMs.

- CE-MS/MS represents an emerging analytical technique able to provide a comprehensive characterization of the primary structure of ADCs. Due to the intrinsic properties of CE-MS/MS, the analysis of an ADC peptide mixture allows the characterization of the amino acid sequence and PTMs with an outstanding robustness, in addition CE-MS/MS analysis enables to locate the conjugated drug on the peptide backbone and estimate the conjugation level independently. As such, CEMS/MS appears as an orthogonal methodology compared to LC-MS/MS peptide mapping for the characterization of ADCs. 


\section{Acknowledgements/ Funding/ Declaration of interest/ CONTACT}

Davy Guillarme wishes to thank the Swiss National Science Foundation for support through a fellowship to Szabolcs Fekete (31003A 159494). Anthony Ehkirch acknowledges the "Association Nationale de la Recherche et de la Technologie" (ANRT) and Syndivia for funding his PhD fellowship. Oscar Hernandez-Alba acknowledges the IdeX program of the University of Strasbourg for funding his postdoctoral fellowship.

\section{ORCID}

Alain Beck: (ID) 0000-0002-4725-1777

Valentina D'Atri (ID): 0000-0002-2601-7092

Sarah Cianferani (ID): 0000-0003-4013-4129

Davy Guillarme (ID): 0000-0001-7883-5823

Yannis Francois (ID): 0000-0002-8776-071X

Rabah Gahoual (ID): 0000-0001-8459-3138

Emmanuelle Leize-Wagner (ID): 0000-0002-1151-7940

Oscar Hernandez-Alba (ID) : 0000-0001-5524-4983

Anthony Ehkirch (ID) : 0000-0002-5616-5280 


\section{References}

Papers of special note have been highlighted as:

- of interest

•• of considerable interest

1. Beck A, Reichert JM. Antibody-drug conjugates. MAbs. 2014;6(1):15-17.

2. Tvito A, Rowe JM. Inotuzumab ozogamicin for the treatment of acute lymphoblastic leukemia. Expert Opin Biol Ther. 2017;17(12):1557-1564.

3. Jen EY, Ko C-W, Lee JE, Del Valle PL, Aydanian A, Jewell C, et al. FDA Approval: Gemtuzumab Ozogamicin for the Treatment of Adults with Newly Diagnosed CD33Positive Acute Myeloid Leukemia. Clin Cancer Res. 2018;24(14):3242-3246.

4. Beck A, Goetsch L, Dumontet C, Corvaïa N. Strategies and challenges for the next generation of antibody-drug conjugates. Nat Rev Drug Discov. 2017;16(5):315-337.

5. Martin C, Kizlik-Masson C, Pèlegrin A, Watier H, Viaud-Massuard M-C, Joubert N. Antibody-drug conjugates: Design and development for therapy and imaging in and beyond cancer, LabEx MAblmprove industrial workshop, July 27-28, 2017, Tours, France. MAbs. 2018;10(2):210-221.

6. Beck A, Jean-Francois H, Thierry W, Liliane G, Christian B, Nathalie C. The next generation of antibody-drug conjugates comes of age. Discov Med. 2010;10(53):329_ 339.

7. Norsworthy KJ, Ko C, Lee JE, Liu J, John CS, Przepiorka D, et al. FDA Approval Summary: Mylotarg for Treatment of Patients with Relapsed or Refractory CD33Positive Acute Myeloid Leukemia. Oncologist. 2018;23(9):1103-1108.

8. Kaplon H, Reichert JM. Antibodies to watch in 2018. MAbs. 2018;10(2):183-203.

9. Kaplon H, Reichert JM. Antibodies to watch in 2019. (In press). MAbs.

10. O'Flaherty R, Trbojević-Akmačić I, Greville G, Rudd PM, Lauc G. The sweet spot for biologics: recent advances in characterization of biotherapeutic glycoproteins. Expert Rev Proteomics. 2018;15(1):13-29.

11. Beck A, Terral G, Debaene F, Wagner-Rousset E, Marcoux J, Janin-Bussat M-C, et al. Cutting-edge mass spectrometry methods for the multi-level structural characterization of antibody-drug conjugates. Expert Rev Proteomics. 2016;13(2):157-183.

12. Liu R, Chen X, Dushime J, Bogalhas M, Lazar AC, Ryll T, et al. The impact of trisulfide 
modification of antibodies on the properties of antibody-drug conjugates manufactured using thiol chemistry. MAbs. 2017;9(3):490-497.

13. Liu-Shin LP-Y, Fung A, Malhotra A, Ratnaswamy G. Evidence of disulfide bond scrambling during production of an antibody-drug conjugate. MAbs. 2018;1-10.

14. Resemann A, Liu-Shin L, Tremintin G, Malhotra A, Fung A, Wang F, et al. Rapid, automated characterization of disulfide bond scrambling and IgG2 isoform determination. MAbs. 2018;1-14.

15. Liu-Shin L, Fung A, Malhotra A, Ratnaswamy G. Influence of disulfide bond isoforms on drug conjugation sites in cysteine-linked IgG2 antibody-drug conjugates. MAbs. 2018;10(4):583-595.

16. Gong HH, Ihle N, Jones MT, Kelly K, Kott L, Raglione T, et al. Control Strategy for Small Molecule Impurities in Antibody-Drug Conjugates. AAPS PharmSciTech. 2018. doi:10.1208/s12249-017-0943-6.

17. Beck A, Wurch T, Bailly C, Corvaia N. Strategies and challenges for the next generation of therapeutic antibodies. Nat Rev Immunol. 2010;10(5):345-352.

18. Rathore D, Faustino A, Schiel J, Pang E, Boyne M, Rogstad S. The role of mass spectrometry in the characterization of biologic protein products. Expert Rev Proteomics. 2018;15(5):431-449.

19. Birdsall RE, McCarthy SM, Janin-Bussat MC, Perez M, Haeuw J-F, Chen W, et al. A sensitive multidimensional method for the detection, characterization, and quantification of trace free drug species in antibody-drug conjugate samples using mass spectral detection. MAbs. 2016;8(2):306-317.

20. Zmolek W, Bañas S, Barfield RM, Rabuka D, Drake PM. A simple LC/MRM-MS-based method to quantify free linker-payload in antibody-drug conjugate preparations. $J$ Chromatogr B. 2016;1032:144-148.

21. Li Y, Gu C, Gruenhagen J, Yehl P, Chetwyn NP, Medley CD. An enzymatic deconjugation method for the analysis of small molecule active drugs on antibody-drug conjugates. MAbs. 2016;8(4):698-705.

22. Friese O V., Smith JN, Brown PW, Rouse JC. Practical approaches for overcoming challenges in heightened characterization of antibody-drug conjugates with new methodologies and ultrahigh-resolution mass spectrometry. MAbs. 2018;10(3):335345. 
23. Neupane R, Bergquist J. Analytical techniques for the characterization of Antibody Drug Conjugates: Challenges and prospects. Eur J Mass Spectrom. 2017;23(6):417-426.

24. Wagh A, Song H, Zeng M, Tao L, Das TK. Challenges and new frontiers in analytical characterization of antibody-drug conjugates. MAbs. 2018;10(2):222-243.

25. Todoroki K, Yamada T, Mizuno H, Toyo'oka T. Current Mass Spectrometric Tools for the Bioanalyses of Therapeutic Monoclonal Antibodies and Antibody-Drug Conjugates. Anal Sci. 2018;34(4):397-406.

26. Chen T, Chen Y, Stella C, Medley CD, Gruenhagen JA, Zhang K. Antibody-drug conjugate characterization by chromatographic and electrophoretic techniques. $J$ Chromatogr B. 2016;1032:39-50.

27. Narhi LO, Schmit J, Bechtold-Peters K, Sharma D. Classification of Protein Aggregates1. J Pharm Sci. 2012;101(2):493-498.

28. Parenky A, Myler H, Amaravadi L, Bechtold-Peters K, Rosenberg A, Kirshner S, et al. New FDA Draft Guidance on Immunogenicity. AAPS J. 2014;16(3):499-503.

29. Fekete S, Beck A, Veuthey J, Guillarme D. Theory and practice of size exclusion chromatography for the analysis of protein aggregates. J Pharm Biomed Anal. 2014;101:161-73.

30. Goyon A, Fekete S, Beck A, Veuthey J-L, Guillarme D. Unraveling the mysteries of modern size exclusion chromatography - the way to achieve confident characterization of therapeutic proteins. J Chromatogr B. 2018;1092:368-378.

31. De Vos J, Kaal ER, Swart R, Baca M, Heyden Y Vander, Eeltink S. Aqueous sizeexclusion chromatographic separations of intact proteins under native conditions: Effect of pressure on selectivity and efficiency. J Sep Sci. 2016;39(4):689-695.

32. Goyon A, Beck A, Veuthey J-L, Guillarme D, Fekete S. Comprehensive study on the effects of sodium and potassium additives in size exclusion chromatographic separations of protein biopharmaceuticals. J Pharm Biomed Anal. 2016. doi:10.1016/j.jpba.2016.09.031.

33. Wakankar A, Chen Y, Gokarn Y, Jacobson FS. Analytical methods for physicochemical characterization of antibody drug conjugates. MAbs. 2011;3(2):161-172.

34. Goyon A, Beck A, Colas O, Sandra K, Guillarme D, Fekete S. Evaluation of size exclusion chromatography columns packed with sub-3 $\mu \mathrm{m}$ particles for the analysis of biopharmaceutical proteins. J Chromatogr A. 2017;1498:80-89. 
35. Hong P, Koza S, Bouvier ESP. Size-Exclusion Chromatography for the Analysis of Protein Biotherapeutics and their Aggregates. J Liq Chromatogr Relat Technol. 2012;35(20):2923-2950.

36. Goyon A, D'Atri V, Colas O, Fekete S, Beck A, Guillarme D. Characterization of 30 therapeutic antibodies and related products by size exclusion chromatography: Feasibility assessment for future mass spectrometry hyphenation. J Chromatogr B Anal Technol Biomed Life Sci. 2017;1065-1066. doi:10.1016/j.jchromb.2017.09.027.

37. Patel A, Gupta V, Hickey J, Nightlinger NS, Rogers RS, Siska C, et al. Coformulation of Broadly Neutralizing Antibodies 3BNC117 and PGT121: Analytical Challenges During Preformulation Characterization and Storage Stability Studies. J Pharm Sci. 2018;107(12):3032-3046.

38. Yang R, Tang Y, Zhang B, Lu X, Liu A, Zhang YT. High resolution separation of recombinant monoclonal antibodies by size-exclusion ultra-high performance liquid chromatography (SE-UHPLC). J Pharm Biomed Anal. 2015;109:52-61.

39. Fekete S, Ganzler K, Guillarme D. Critical evaluation of fast size exclusion chromatographic separations of protein aggregates, applying sub-2 $\mu \mathrm{m}$ particles. $\mathrm{J}$ Pharm Biomed Anal. 2013;78-79:141-149.

40. Goyon A, Guillarme D, Fekete S. The importance of system band broadening in modern size exclusion chromatography. J Pharm Biomed Anal. 2017;135:50-60.

41. Rodriguez-Aller M, Guillarme D, Beck A, Fekete S. Practical method development for the separation of monoclonal antibodies and antibody-drug-conjugate species in hydrophobic interaction chromatography, part 1: optimization of the mobile phase. $\mathrm{J}$ Pharm Biomed Anal. 2016;118:393-403.

42. Cusumano A, Guillarme D, Beck A, Fekete S. Practical method development for the separation of monoclonal antibodies and antibody-drug-conjugate species in hydrophobic interaction chromatoraphy, part 2: Optimization of the phase system. $J$ Pharm Biomed Anal. 2016;121:161-173.

43. Bobaly B, Beck A, Veuthey J-L, Guillarme D, Fekete S. Impact of organic modifier and temperature on protein denaturation in hydrophobic interaction chromatography. $J$ Pharm Biomed Anal. 2016;131:124-132.

44. Chen B, Peng Y, Valeja SG, Xiu L, Alpert AJ, Ge Y. Online Hydrophobic Interaction Chromatography-Mass Spectrometry for Top-Down Proteomics. Anal Chem. 2016;88(3):1885-1891. 
45. Bobály B, Randazzo GM, Rudaz S, Guillarme D, Fekete S. Optimization of non-linear gradient in hydrophobic interaction chromatography for the analytical characterization of antibody-drug conjugates. J Chromatogr A. 2017;1481:82-91.

46. Fekete S, Beck A, Veuthey J-L, Guillarme D. lon-exchange chromatography for the characterization of biopharmaceuticals. J Pharm Biomed Anal. 2015;113:43-55.

47. Fekete S, Beck A, Fekete J, Guillarme D. Method development for the separation of monoclonal antibody charge variants in cation exchange chromatography, Part I: Salt gradient approach. J Pharm Biomed Anal. 2015;102:33-44.

48. Lau H, Pace D, Yan B, McGrath T, Smallwood S, Patel K, et al. Investigation of degradation processes in $\lg \mathrm{G} 1$ monoclonal antibodies by limited proteolysis coupled with weak cation-exchange HPLC. J Chromatogr B. 2010;878(11-12):868-876.

49. Goyon A, Sciascera L, Clarke A, Guillarme D, Pell R. Extending the limits of size exclusion chromatography: Simultaneous separation of free payloads and related species from antibody drug conjugates and their aggregates. $J$ Chromatogr $A$. 2018;1539:19-29.

50. Xiaoying Chen, Nguyen M, Jacobson F, Ouyang J. Charge-based analysis of antibodies with engineered cysteines. MAbs. 2009;1(6):563-571.

51. Boylan NJ, Zhou W, Proos RJ, Tolbert TJ, Wolfe JL, Laurence JS. Conjugation Site Heterogeneity Causes Variable Electrostatic Properties in Fc Conjugates. Bioconjug Chem. 2013;24(6):1008-1016.

52. Fekete S, Beck A, Wagner E, Vuignier K, Guillarme D. Adsorption and recovery issues of recombinant monoclonal antibodies in reversed-phase liquid chromatography. J Sep Sci. 2015;38(1):1-8.

53. Fekete S, Guillarme D, Sandra P, Sandra K. Chromatographic, Electrophoretic, and Mass Spectrometric Methods for the Analytical Characterization of Protein Biopharmaceuticals. Anal Chem. 2016;88(1):480-507.

54. Bobály B, Fleury-Souverain S, Beck A, Veuthey J-L, Guillarme D, Fekete S. Current possibilities of liquid chromatography for the characterization of antibody-drug conjugates. J Pharm Biomed Anal. 2018;147:493-505.

55. Bobály B, Lauber M, Beck A, Guillarme D, Fekete S. Utility of a high coverage phenylbonding and wide-pore superficially porous particle for the analysis of monoclonal antibodies and related products. J Chromatogr A. 2018;1549:63-76. 
56. Bobály B, D'Atri V, Lauber M, Beck A, Guillarme D, Fekete S. Characterizing various monoclonal antibodies with milder reversed phase chromatography conditions. J Chromatogr B Anal Technol Biomed Life Sci. 2018;1096(March):1-10.

57. Fekete S, Molnár I, Guillarme D. Separation of antibody drug conjugate species by RPLC: A generic method development approach. J Pharm Biomed Anal. 2017;137:6069.

58. Marcoux J, Champion T, Colas O, Wagner-Rousset E, Corvaïa N, Van Dorsselaer A, et al. Native mass spectrometry and ion mobility characterization of trastuzumab emtansine, a lysine-linked antibody drug conjugate. Protein Sci. 2015;24(8):1210-1223.

59. Xu Y, Jiang G, Tran C, Li X, Heibeck TH, Masikat MR, et al. RP-HPLC DAR Characterization of Site-Specific Antibody Drug Conjugates Produced in a Cell-Free Expression System. Org Process Res Dev. 2016;20(6):1034-1043.

60. D'Atri V, Pell R, Clarke A, Guillarme D, Fekete S. Is hydrophobic interaction chromatography the most suitable technique to characterize site-specific antibody-drug conjugates? Submitted to. J Chromatogr B.

61. Periat A, Fekete S, Cusumano A, Veuthey J-L, Beck A, Lauber M, et al. Potential of hydrophilic interaction chromatography for the analytical characterization of protein biopharmaceuticals. J Chromatogr A. 2016;1448:81-92.

62. Bobály B, D'Atri V, Beck A, Guillarme D, Fekete S. Analysis of recombinant monoclonal antibodies in hydrophilic interaction chromatography: A generic method development approach. J Pharm Biomed Anal. 2017;145:24-32.

63. D'Atri V, Fekete S, Stoll D, Lauber M, Beck A, Guillarme D. Characterization of an antibody-drug conjugate by hydrophilic interaction chromatography coupled to mass spectrometry. J Chromatogr B Anal Technol Biomed Life Sci. 2018;1080. doi:10.1016/j.jchromb.2018.02.026.

64. D'Atri V, Fekete S, Beck A, Lauber M, Guillarme D. Hydrophilic Interaction Chromatography Hyphenated with Mass Spectrometry: A Powerful Analytical Tool for the Comparison of Originator and Biosimilar Therapeutic Monoclonal Antibodies at the Middle-up Level of Analysis. Anal Chem. 2017;89(3):2086-2092.

65. D'Atri V, Dumont E, Vandenheede I, Guillarme D, Sandra P, Sandra K. Hydrophilic interaction chromatography for the characterization of therapeutic monoclonal antibodies at protein, peptide, and glycan levels. LC-GC Eur. 2017;30(8).

66. Wang S, Liu AP, Yan Y, Daly TJ, Li N. Characterization of product-related low molecular 
weight impurities in therapeutic monoclonal antibodies using hydrophilic interaction chromatography coupled with mass spectrometry. J Pharm Biomed Anal. 2018;154:468-475.

67. Stoll DR, Harmes DC, Staples GO, Potter OG, Dammann CT, Guillarme D, et al. Development of Comprehensive Online Two-Dimensional Liquid Chromatography/Mass Spectrometry Using Hydrophilic Interaction and ReversedPhase Separations for Rapid and Deep Profiling of Therapeutic Antibodies. Anal Chem. 2018;90(9):5923-5929.

68. Hu S, Dovichi NJ. Capillary Electrophoresis for the Analysis of Biopolymers. Anal Chem. 2002;74(12):2833-2850.

69. Zhang Z, Qu Y, Dovichi NJ. Capillary zone electrophoresis-mass spectrometry for bottom-up proteomics. TrAC Trends Anal Chem. 2018;108:23-37.

70. Gahoual R, Giorgetti J, Beck A, Leize-Wagner E, François Y-N. Biopharmaceutical Applications of Capillary Electromigration Methods. In: Capillary Electromigration Separation Methods. 2018. Elsevier: 453-480.

71. Gahoual R, Beck A, Leize-Wagner E, François Y-N. Cutting-edge capillary electrophoresis characterization of monoclonal antibodies and related products. $J$ Chromatogr B. 2016;1032:61-78.

72. Zhu Z, Lu JJ, Liu S. Protein separation by capillary gel electrophoresis: A review. Anal Chim Acta. 2012;709:21-31.

73. Goyon A, Excoffier M, Janin Bussat MC, Bobaly B, Fekete S, Guillarme D, et al. Determination of isoelectric points and relative charge variants of 23 therapeutic monoclonal antibodies. J Chromatogr B. 2017;1066(September):119-128.

74. Goyon A, Francois YN, Colas O, Beck A, Veuthey JL, Guillarme D. High-resolution separation of monoclonal antibodies mixtures and their charge variants by an alternative and generic CZE method. Electrophoresis. 2018;39(16):2083-2090.

75. Giorgetti J, D'Atri V, Canonge J, Lechner A, Guillarme D, Colas O, et al. Monoclonal antibody $\mathrm{N}$-glycosylation profiling using capillary electrophoresis - Mass spectrometry: Assessment and method validation. Talanta. 2018;178. doi:10.1016/j.talanta.2017.09.083.

76. Gahoual R, Beck A, François Y-N, Leize-Wagner E. Independent highly sensitive characterization of asparagine deamidation and aspartic acid isomerization by sheathless CZE-ESI-MS/MS. J Mass Spectrom. 2016;51(2):150-158. 
77. Szekrényes Á, Park SS, Santos M, Lew C, Jones A, Haxo T, et al. Multi-Site N-glycan mapping study 1: Capillary electrophoresis - laser induced fluorescence. MAbs. 2016;8(1):56-64.

78. Salas-Solano O, Babu K, Park SS, Zhang X, Zhang L, Sosic Z, et al. Intercompany Study to Evaluate the Robustness of Capillary Isoelectric Focusing Technology for the Analysis of Monoclonal Antibodies. Chromatographia. 2011;73(11-12):1137-1144.

79. Wu G, Yu C, Wang W, Wang L. Interlaboratory method validation of iclEF methodology for analysis of monoclonal antibodies. Electrophoresis. 2018;39(16):2091-2098.

80. Moritz B, Schnaible V, Kiessig S, Heyne A, Wild M, Finkler C, et al. Evaluation of capillary zone electrophoresis for charge heterogeneity testing of monoclonal antibodies. J Chromatogr B. 2015;983-984:101-110.

81. Liu JP, Abid S, Lee MS. Analysis of Monoclonal Antibody Chimeric BR96-Doxorubicin Immunoconjugate by Sodium Dodecyl Sulfate-Capillary Gel Electrophoresis with Ultraviolet and Laser-Induced Fluorescence Detection. Anal Biochem. 1995;229(2):221-228.

82. Sun MMC, Beam KS, Cerveny CG, Hamblett KJ, Blackmore RS, Torgov MY, et al. Reduction-Alkylation Strategies for the Modification of Specific Monoclonal Antibody Disulfides. Bioconjug Chem. 2005;16(5):1282-1290.

83. Le LN, Moore JMR, Ouyang J, Chen X, Nguyen MDH, Galush WJ. Profiling Antibody Drug Conjugate Positional Isomers: A System-of-Equations Approach. Anal Chem. 2012;84(17):7479-7486.

84. Chen L, Wang L, Shion H, Yu C, Yu YQ, Zhu L, et al. In-depth structural characterization of Kadcyla® (ado-trastuzumab emtansine) and its biosimilar candidate. MAbs. 2016;8(7):1210-1223.

85. Felten C, Salas-Solano O, Michels DA. Imaged Capillary Isoelectric Focusing for Charge-Variant Analysis of Biopharmaceuticals. Bioprocess Int. 2011;9:48-53.

86. Ji JA, Liu J, Wang J. Formulation development for Antibody-Drug Conjugates. In: Wang J, Shen W-C, Zaro JL, editors. Antibody-Drug Conjugates. 2015. Cham. Springer International Publishing: 79-95.

87. Adem YT, Schwarz KA, Duenas E, Patapoff TW, Galush WJ, Esue O. Auristatin Antibody Drug Conjugate Physical Instability and the Role of Drug Payload. Bioconjug Chem. 2014;25(4):656-664. 
88. Valliere-Douglass JF, Lewis P, Salas-Solano O, Jiang S. Solid-State mAbs and ADCs Subjected to Heat-Stress Stability Conditions can be Covalently Modified with Buffer and Excipient Molecules. J Pharm Sci. 2015;104(2):652-665.

89. Jeffrey SC, Burke PJ, Lyon RP, Meyer DW, Sussman D, Anderson M, et al. A Potent Anti-CD70 Antibody-Drug Conjugate Combining a Dimeric Pyrrolobenzodiazepine Drug with Site-Specific Conjugation Technology. Bioconjug Chem. 2013;24(7):1256-1263.

90. Lin J, Lazar AC. Detremination of charge heterogeneity and level of unconjugated antibody by imaged iCIEF. In: Ducry L, editor. Antibody-Drug Conjugates. 2013. Totowa, NJ. Humana Press: 295-302.

91. Labrijn AF, Buijsse AO, van den Bremer ETJ, Verwilligen AYW, Bleeker WK, Thorpe SJ, et al. Therapeutic lgG4 antibodies engage in Fab-arm exchange with endogenous human IgG4 in vivo. Nat Biotechnol. 2009;27(8):767-771.

92. Maeda E, Urakami K, Shimura K, Kinoshita M, Kakehi K. Charge heterogeneity of a therapeutic monoclonal antibody conjugated with a cytotoxic antitumor antibiotic, calicheamicin. J Chromatogr A. 2010;1217(45):7164-7171.

93. Luo $\mathrm{Q}$, Chung $\mathrm{HH}$, Borths $\mathrm{C}$, Janson $\mathrm{M}$, Wen J, Joubert $\mathrm{MK}$, et al. Structural Characterization of a Monoclonal Antibody-Maytansinoid Immunoconjugate. Anal Chem. 2016;88(1):695-702.

94. Liu J, Zhao H, Volk KJ, Klohr SE, Kerns EH, Lee MS. Analysis of monoclonal antibody and immunoconjugate digests by capillary electrophoresis and capillary liquid chromatography. J Chromatogr A. 1996;735(1-2):357-366.

95. Kubota K, Kobayashi N, Yabuta M, Ohara M, Naito T, Kubo T, et al. Validation of Capillary Zone Electrophoretic Method for Evaluating Monoclonal Antibodies and Antibody-Drug Conjugates. Chromatography. 2016;37(3):117-124.

96. Henley WH, He Y, Mellors JS, Batz NG, Ramsey JM, Jorgenson JW. High resolution separations of charge variants and disulfide isomers of monoclonal antibodies and antibody drug conjugates using ultra-high voltage capillary electrophoresis with high electric field strength. J Chromatogr A. 2017;1523:72-79.

97. Lössl P, van de Waterbeemd M, Heck AJ. The diverse and expanding role of mass spectrometry in structural and molecular biology. EMBO J. 2016;35(24):2634-2657.

98. Eschweiler JD, Kerr R, Rabuck-Gibbons J, Ruotolo BT. Sizing Up Protein-Ligand Complexes: The Rise of Structural Mass Spectrometry Approaches in the Pharmaceutical Sciences. Annu Rev Anal Chem. 2017;10(1):25-44. 
99. Pedro L, Quinn R. Native Mass Spectrometry in Fragment-Based Drug Discovery. Molecules. 2016;21(8):984.

100. Beck A, Sanglier-Cianférani S, Van Dorsselaer A. Biosimilar, Biobetter, and Next Generation Antibody Characterization by Mass Spectrometry. Anal Chem. 2012;84(11):4637-4646.

101. Beck A, Wagner-Rousset E, Ayoub D, Van Dorsselaer A, Sanglier-Cianférani S. Characterization of Therapeutic Antibodies and Related Products. Anal Chem. 2013;85(2):715-736.

102. Beck A, Debaene F, Diemer H, Wagner-Rousset E, Colas O, Dorsselaer A Van, et al. Cutting-edge mass spectrometry characterization of originator, biosimilar and biobetter antibodies. J Mass Spectrom. 2015;50(2):285-297.

103. Zhang $\mathrm{H}$, Cui W, Gross ML. Mass spectrometry for the biophysical characterization of therapeutic monoclonal antibodies. FEBS Lett. 2014;588(2):308-317.

104. Debaene F, Wagner-Rousset E, Colas O, Ayoub D, Corvaia N, Van Dorsselaer A, et al. Time Resolved Native Ion-Mobility Mass Spectrometry to Monitor Dynamics of IgG4 Fab Arm Exchange and 'Bispecific' Monoclonal Antibody Formation. Anal Chem. 2013;85(20):9785-9792.

105. Rosati S, Yang Y, Barendregt A, Heck AJR. Detailed mass analysis of structural heterogeneity in monoclonal antibodies using native mass spectrometry. Nat Protoc. 2014;9(4):967-976.

106. Beck A, Diemer H, Ayoub D, Debaene F, Wagner-Rousset E, Carapito C, et al. Analytical characterization of biosimilar antibodies and Fc-fusion proteins. TrAC Trends Anal Chem. 2013;48:81-95.

107. Rosati S, Rose RJ, Thompson NJ, van Duijn E, Damoc E, Denisov E, et al. Exploring an Orbitrap Analyzer for the Characterization of Intact Antibodies by Native Mass Spectrometry. Angew Chemie Int Ed. 2012;51(52):12992-12996.

108. Thompson NJ, Hendriks LJ, de Kruif J, Throsby M, Heck AJ. Complex mixtures of antibodies generated from a single production qualitatively and quantitatively evaluated by native Orbitrap mass spectrometry. MAbs. 2014;6(1):197-203.

109. Thompson NJ, Rosati S, Heck AJR. Performing native mass spectrometry analysis on therapeutic antibodies. Methods. 2014;65(1):11-17.

110. Valliere-Douglass JF, McFee WA, Salas-Solano O. Native Intact Mass Determination of 
Antibodies Conjugated with Monomethyl Auristatin E and F at Interchain Cysteine Residues. Anal Chem. 2012;84(6):2843-2849.

111. Debaene F, Bœuf A, Wagner-Rousset E, Colas O, Ayoub D, Corvaïa N, et al. Innovative Native MS Methodologies for Antibody Drug Conjugate Characterization: High Resolution Native MS and IM-MS for Average DAR and DAR Distribution Assessment. Anal Chem. 2014;86(21):10674-10683.

112. Rosati S, Van Den Bremer ET, Schuurman J, Parren PW, Kamerling JP, Heck AJ. Indepth qualitative and quantitative analysis of composite glycosylation profiles and other micro-heterogeneity on intact monoclonal antibodies by high-resolution native mass spectrometry using a modified Orbitrap. MAbs. 2013;5(6):917-924.

113. Tian Y, Ruotolo BT. The growing role of structural mass spectrometry in the discovery and development of therapeutic antibodies. Analyst. 2018;143(11):2459-2468.

114. Göth M, Pagel K. Ion mobility-mass spectrometry as a tool to investigate protein-ligand interactions. Anal Bioanal Chem. 2017;409(18):4305-4310.

115. Ben-Nissan G, Sharon M. The application of ion-mobility mass spectrometry for structure/function investigation of protein complexes. Curr Opin Chem Biol. 2018;42:2533.

116. Hengel SM, Sanderson R, Valliere-Douglass J, Nicholas N, Leiske C, Alley SC. Measurement of in Vivo Drug Load Distribution of Cysteine-Linked Antibody-Drug Conjugates Using Microscale Liquid Chromatography Mass Spectrometry. Anal Chem. 2014;86(7):3420-3425.

117. Chen J, Yin S, Wu Y, Ouyang J. Development of a Native Nanoelectrospray Mass Spectrometry Method for Determination of the Drug-to-Antibody Ratio of Antibody-Drug Conjugates. Anal Chem. 2013;85(3):1699-1704.

118. Dovgan I, Ursuegui S, Erb S, Michel C, Kolodych S, Cianférani S, et al. Acyl Fluorides: Fast, Efficient, and Versatile Lysine-Based Protein Conjugation via Plug-and-Play Strategy. Bioconjug Chem. 2017;28(5):1452-1457.

119. Koniev O, Dovgan I, Renoux B, Ehkirch A, Eberova J, Cianférani S, et al. Reductionrebridging strategy for the preparation of ADPN-based antibody-drug conjugates. Medchemcomm. 2018;9(5):827-830.

120. Botzanowski T, Erb S, Hernandez-Alba O, Ehkirch A, Colas O, Wagner-Rousset E, et al. Insights from native mass spectrometry approaches for top- and middle- level characterization of site-specific antibody-drug conjugates. MAbs. 2017;9(5):801-811. 
121. Ehkirch A, Hernandez-Alba O, Colas O, Beck A, Guillarme D, Cianférani S. Hyphenation of size exclusion chromatography to native ion mobility mass spectrometry for the analytical characterization of therapeutic antibodies and related products. J Chromatogr B Anal Technol Biomed Life Sci. 2018;1086(March):176-183.

122. Bern M, Caval T, Kil YJ, Tang W, Becker C, Carlson E, et al. Parsimonious Charge Deconvolution for Native Mass Spectrometry. J Proteome Res. 2018;17(3):1216-1226.

123. Campuzano IDG, Netirojjanakul C, Nshanian M, Lippens JL, Kilgour DPA, Van Orden $S$, et al. Native-MS Analysis of Monoclonal Antibody Conjugates by Fourier Transform Ion Cyclotron Resonance Mass Spectrometry. Anal Chem. 2018;90(1):745-751.

124. Muneeruddin K, Nazzaro M, Kaltashov IA. Characterization of Intact Protein Conjugates and Biopharmaceuticals Using lon-Exchange Chromatography with Online Detection by Native Electrospray Ionization Mass Spectrometry and Top-Down Tandem Mass Spectrometry. Anal Chem. 2015;87(19):10138-10145.

125. Muneeruddin K, Thomas JJ, Salinas PA, Kaltashov IA. Characterization of Small Protein Aggregates and Oligomers Using Size Exclusion Chromatography with Online Detection by Native Electrospray Ionization Mass Spectrometry. Anal Chem. 2014;86(21):10692-10699.

126. Belov AM, Viner R, Santos MR, Horn DM, Bern M, Karger BL, et al. Analysis of Proteins, Protein Complexes, and Organellar Proteomes Using Sheathless Capillary Zone Electrophoresis - Native Mass Spectrometry. J Am Soc Mass Spectrom. 2017;28(12):2614-2634.

127. Fekete S, Veuthey J-L, Beck A, Guillarme D. Hydrophobic interaction chromatography for the characterization of monoclonal antibodies and related products. J Pharm Biomed Anal. 2016;130:3-18.

128. Griaud F, Denefeld B, Lang M, Hensinger H, Haberl P, Berg M. Unbiased in-depth characterization of CEX fractions from a stressed monoclonal antibody by mass spectrometry. MAbs. 2017;9(5):820-830.

129. Sorensen M, Harmes DC, Stoll DR, Staples GO, Fekete S, Guillarme D, et al. Comparison of originator and biosimilar therapeutic monoclonal antibodies using comprehensive two-dimensional liquid chromatography coupled with time-of-flight mass spectrometry. MAbs. 2016;8(7):1224-1234.

130. Stoll DR, Harmes DC, Danforth J, Wagner E, Guillarme D, Fekete S, et al. Direct Identification of Rituximab Main Isoforms and Subunit Analysis by Online Selective 
Comprehensive Two-Dimensional Liquid Chromatography-Mass Spectrometry. Anal Chem. 2015;87(16):8307-8315.

131. Sarrut M, Corgier A, Fekete S, Guillarme D, Lascoux D, Janin-Bussat M-C, et al. Analysis of antibody-drug conjugates by comprehensive on-line two-dimensional hydrophobic interaction chromatography $x$ reversed phase liquid chromatography hyphenated to high resolution mass spectrometry. I - Optimization of separation conditions. J Chromatogr B. 2016;1032:103-111.

132. Muneeruddin K, Bobst CE, Frenkel R, Houde D, Turyan I, Sosic Z, et al. Characterization of a PEGylated protein therapeutic by ion exchange chromatography with on-line detection by native ESI MS and MS/MS. Analyst. 2017;142(2):336-344.

133. Talebi M, Nordborg A, Gaspar A, Lacher NA, Wang Q, He XZ, et al. Charge heterogeneity profiling of monoclonal antibodies using low ionic strength ion-exchange chromatography and well-controlled pH gradients on monolithic columns. J Chromatogr A. 2013;1317:148-154.

134. Leblanc Y, Ramon C, Bihoreau N, Chevreux G. Charge variants characterization of a monoclonal antibody by ion exchange chromatography coupled on-line to native mass spectrometry: Case study after a long-term storage at $+5^{\circ} \mathrm{C}$. J Chromatogr B Anal Technol Biomed Life Sci. 2017;1048:130-139.

135. FüssI F, Trappe A, Cook K, Scheffler K, Fitzgerald O, Bones J. Comprehensive characterisation of the heterogeneity of adalimumab via charge variant analysis hyphenated on-line to native high resolution Orbitrap mass spectrometry. MAbs. 2018;1-13.

136. FüssI F, Cook K, Scheffler K, Farrell A, Mittermayr S, Bones J. Charge Variant Analysis of Monoclonal Antibodies Using Direct Coupled pH Gradient Cation Exchange Chromatography to High-Resolution Native Mass Spectrometry. Anal Chem. 2018;90(7):4669-4676.

137. Bailey AO, Han G, Phung W, Gazis P, Sutton J, Josephs JL, et al. Charge variant native mass spectrometry benefits mass precision and dynamic range of monoclonal antibody intact mass analysis. MAbs. 2018;1-12.

138. Chen B, Lin Z, Alpert AJ, Fu C, Zhang Q, Pritts WA, et al. Online Hydrophobic Interaction Chromatography - Mass Spectrometry for the Analysis of Intact Monoclonal Antibodies. Anal Chem. 2018;90:8-11.

139. D’Atri V, Causon T, Hernandez-Alba O, Mutabazi A, Veuthey J-L, Cianferani S, et al. 
Adding a new separation dimension to MS and LC-MS: What is the utility of ion mobility spectrometry? J Sep Sci. 2018;41(1):20-67.

140. Hernandez-Alba O, Wagner-Rousset E, Beck A, Cianférani S. Native Mass Spectrometry, Ion Mobility and Collision Induced Unfolding for Conformational Characterization of IgG4 Monoclonal Antibodies. Anal Chem. 2018. doi:10.1021/acs.analchem.8b00912.

141. Devine PWA, Fisher HC, Calabrese AN, Whelan F, Higazi DR, Potts JR, et al. Investigating the Structural Compaction of Biomolecules Upon Transition to the GasPhase Using ESI-TWIMS-MS. J Am Soc Mass Spectrom. 2017;1-8.

142. Ruotolo BT, Benesch JLP, Sandercock AM, Hyung S-J, Robinson C V. Ion mobilitymass spectrometry analysis of large protein complexes. Nat Protoc. 2008;3(7):1139_ 1152.

143. Hansen K, Lau AMC, Giles K, McDonnell JM, Struwe WB, Sutton BJ, et al. A mass spectrometry-based modelling workflow for accurate prediction of $\lg G$ antibody conformations in the gas phase. Angew Chemie Int Ed. 2018. doi:10.1002/anie.201812018.

144. Pacholarz KJ, Porrini M, Garlish RA, Burnley RJ, Taylor RJ, Henry AJ, et al. Dynamics of Intact Immunoglobulin-G Explored by Drift-Tube Ion-Mobility Mass Spectrometry and Molecular Modeling. Angew Chemie Int Ed. 2014;53(30):7765-7769.

145. Tian Y, Han L, Buckner AC, Ruotolo BT. Collision Induced Unfolding of Intact Antibodies: Rapid Characterization of Disulfide Bonding Patterns, Glycosylation, and Structures. Anal Chem. 2015;87(22):11509-11515.

146. Watanabe Y, Vasiljevic S, Allen JD, Seabright GE, Duyvesteyn HME, Doores KJ, et al. Signature of Antibody Domain Exchange by Native Mass Spectrometry and CollisionInduced Unfolding. Anal Chem. 2018;90(12):7325-7331.

147. Ferguson CN, Gucinski-Ruth AC. Evaluation of Ion Mobility-Mass Spectrometry for Comparative Analysis of Monoclonal Antibodies. J Am Soc Mass Spectrom. 2016;27(5):822-833.

148. Pisupati K, Tian Y, Okbazghi S, Benet A, Ackermann R, Ford M, et al. A Multidimensional Analytical Comparison of Remicade and the Biosimilar Remsima. Anal Chem. 2017;89(9):4838-4846.

149. Migas LG, France AP, Bellina B, Barran PE. ORIGAMI : A software suite for activated ion mobility mass spectrometry (aIM-MS) applied to multimeric protein assemblies. Int 
J Mass Spectrom. 2018;427:20-28.

150. Eschweiler JD, Rabuck-Gibbons JN, Tian Y, Ruotolo BT. CIUSuite: A Quantitative Analysis Package for Collision Induced Unfolding Measurements of Gas-Phase Protein lons. Anal Chem. 2015;87(22):11516-11522.

151. Janin-Bussat $M-C$, Dillenbourg $M$, Corvaia N, Beck A, Klinguer-Hamour C. Characterization of antibody drug conjugate positional isomers at cysteine residues by peptide mapping LC-MS analysis. J Chromatogr B. 2015;981-982:9-13.

152. Zhang Z, Pan H, Chen X. Mass spectrometry for structural characterization of therapeutic antibodies. Mass Spectrom Rev. 2009;28(1):147-176.

153. Mitchell Wells J, McLuckey SA. Collision- Induced Dissociation (CID) of Peptides and Proteins. 2005: 148-185.

154. Fornelli L, Ayoub D, Aizikov K, Liu X, Damoc E, Pevzner PA, et al. Top-down analysis of immunoglobulin $\mathrm{G}$ isotypes 1 and 2 with electron transfer dissociation on a high-field Orbitrap mass spectrometer. J Proteomics. 2017;159:67-76.

155. Tsybin YO, Fornelli L, Stoermer C, Luebeck M, Parra J, Nallet S, et al. Structural Analysis of Intact Monoclonal Antibodies by Electron Transfer Dissociation Mass Spectrometry. Anal Chem. 2011;83(23):8919-8927.

156. He L, Anderson LC, Barnidge DR, Murray DL, Hendrickson CL, Marshall AG. Erratum to: Analysis of Monoclonal Antibodies in Human Serum as a Model for Clinical Monoclonal Gammopathy by Use of 21 Tesla FT-ICR Top-Down and Middle-Down MS/MS. J Am Soc Mass Spectrom. 2017;28(5):839-839.

157. Fornelli L, Damoc E, Thomas PM, Kelleher NL, Aizikov K, Denisov E, et al. Analysis of Intact Monoclonal Antibody lgG1 by Electron Transfer Dissociation Orbitrap FTMS. Mol Cell Proteomics. 2012;11(12):1758-1767.

158. Mao Y, Valeja SG, Rouse JC, Hendrickson CL, Marshall AG. Top-Down Structural Analysis of an Intact Monoclonal Antibody by Electron Capture Dissociation-Fourier Transform Ion Cyclotron Resonance-Mass Spectrometry. Anal Chem. 2013;85(9):4239-4246.

159. Fornelli L, Srzentić K, Huguet R, Mullen C, Sharma S, Zabrouskov V, et al. Accurate Sequence Analysis of a Monoclonal Antibody by Top-Down and Middle-Down Orbitrap Mass Spectrometry Applying Multiple Ion Activation Techniques. Anal Chem. 2018;90(14):8421-8429. 
160. Cotham VC, Brodbelt JS. Characterization of Therapeutic Monoclonal Antibodies at the Subunit-Level using Middle-Down 193 nm Ultraviolet Photodissociation. Anal Chem. 2016;88(7):4004-4013.

161. Hernandez-Alba O, Houel S, Erb S, Rabuka D, Huguet R, Josephs JL, et al. A comprehensive characterization of a site-specific antibody-drug-conjugate by middledown MS with multiple fragmentation techniques. Submitted to. MAbs.

162. Dyachenko A, Wang G, Belov M, Makarov A, de Jong RN, van den Bremer ETJ, et al. Tandem Native Mass-Spectrometry on Antibody-Drug Conjugates and Submillion Da Antibody-Antigen Protein Assemblies on an Orbitrap EMR Equipped with a High-Mass Quadrupole Mass Selector. Anal Chem. 2015;87(12):6095-6102.

163. Huang RY-C, Chen G. Higher order structure characterization of protein therapeutics by hydrogen/deuterium exchange mass spectrometry. Anal Bioanal Chem. 2014;406(26):6541-6558.

164. Houde D, Arndt J, Domeier W, Berkowitz S, Engen JR. Characterization of IgG1 Conformation and Conformational Dynamics by Hydrogen/Deuterium Exchange Mass Spectrometry. Anal Chem. 2009;81(14):5966-5966.

165. Houde D, Peng Y, Berkowitz SA, Engen JR. Post-translational Modifications Differentially Affect IgG1 Conformation and Receptor Binding. Mol Cell Proteomics. 2010;9(8):1716-1728.

166. lacob RE, Krystek SR, Huang RY-C, Wei H, Tao L, Lin Z, et al. Hydrogen/deuterium exchange mass spectrometry applied to IL-23 interaction characteristics: potential impact for therapeutics. Expert Rev Proteomics. 2015;12(2):159-169.

167. Huang RY-C, Krystek SR, Felix N, Graziano RF, Srinivasan M, Pashine A, et al. Hydrogen/deuterium exchange mass spectrometry and computational modeling reveal a discontinuous epitope of an antibody/TL1A Interaction. MAbs. 2018;10(1):95-103.

168. Terral G, Champion T, Debaene F, Colas O, Bourguet M, Wagner-Rousset E, et al. Epitope characterization of anti-JAM-A antibodies using orthogonal mass spectrometry and surface plasmon resonance approaches. MAbs. 2017;9(8):1317-1326.

169. Pan LY, Salas-Solano O, Valliere-Douglass JF. Conformation and Dynamics of Interchain Cysteine-Linked Antibody-Drug Conjugates as Revealed by Hydrogen/Deuterium Exchange Mass Spectrometry. Anal Chem. 2014;86(5):26572664 .

170. Pan LY, Salas-Solano O, Valliere-Douglass JF. Antibody Structural Integrity of Site- 
Specific Antibody-Drug Conjugates Investigated by Hydrogen/Deuterium Exchange Mass Spectrometry. Anal Chem. 2015;87(11):5669-5676.

171. Huang RY-C, O'Neil SR, Lipovšek D, Chen G. Conformational Assessment of Adnectin and Adnectin-Drug Conjugate by Hydrogen/Deuterium Exchange Mass Spectrometry. J Am Soc Mass Spectrom. 2018;29(7):1524-1531.

172. Pan LY, Salas-Solano O, Valliere-Douglass JF. Localized conformational interrogation of antibody and antibody-drug conjugates by site-specific carboxyl group footprinting. MAbs. 2017;9(2):307-318.

173. Wang $X$, Buckenmaier S, Stoll D. The growing role of two-dimensional LC in the biopharmaceutical industry. J Appl Bioanal. 2017;3(5):120-126.

174. Li Y, Gu C, Gruenhagen J, Zhang K, Yehl P, Chetwyn NP, et al. A size exclusionreversed phase two dimensional-liquid chromatography methodology for stability and small molecule related species in antibody drug conjugates. J Chromatogr A. 2015;1393:81-88.

175. Li Y, Stella C, Zheng L, Bechtel C, Gruenhagen J, Jacobson F, et al. Investigation of low recovery in the free drug assay for antibody drug conjugates by size exclusionreversed phase two dimensional-liquid chromatography. $J$ Chromatogr $B$. 2016;1032:112-118.

176. Vanhoenacker G, Vandenheede I, David F, Sandra P, Sandra K. Comprehensive twodimensional liquid chromatography of therapeutic monoclonal antibody digests. Anal Bioanal Chem. 2015;407(1):355-366.

177. Sarrut M, Fekete S, Janin-Bussat M-C, Colas O, Guillarme D, Beck A, et al. Analysis of antibody-drug conjugates by comprehensive on-line two-dimensional hydrophobic interaction chromatography $\mathrm{x}$ reversed phase liquid chromatography hyphenated to high resolution mass spectrometry. II- Identification of sub-units for the characteri. $J$ Chromatogr B. 2016;1032:91-102.

178. Ehkirch A, D’Atri V, Rouviere F, Hernandez-Alba O, Goyon A, Colas O, et al. An Online Four-Dimensional HIC $\times$ SEC-IM $\times$ MS Methodology for Proof-of-Concept Characterization of Antibody Drug Conjugates. Anal Chem. 2018;90(3):1578-1586.

179. Gahoual R, Busnel J-M, Wolff P, François YN, Leize-Wagner E. Novel sheathless CEMS interface as an original and powerful infusion platform for nanoESI study: from intact proteins to high molecular mass noncovalent complexes. Anal Bioanal Chem. 2014;406(4):1029-1038. 
180. Höcker O, Montealegre C, Neusüß C. Characterization of a nanoflow sheath liquid interface and comparison to a sheath liquid and a sheathless porous-tip interface for CE-ESI-MS in positive and negative ionization. Anal Bioanal Chem. 2018;410(21):5265-5275.

181. Said N, Giorgetti J, Kuhn L, Beck A, Leize-wagner E, Gahoual R, et al. Advanced Antibody-Drug Conjugate Structural Characterization by Sheathless Capillary Electrophoresis-Tandem Mass Spectrometry Using Complementary Approaches. LCGC North Am. 2017;15(1):15-21.

182. Said N, Gahoual R, Kuhn L, Beck A, François Y-N, Leize-Wagner E. Structural characterization of antibody drug conjugate by a combination of intact, middle-up and bottom-up techniques using sheathless capillary electrophoresis - Tandem mass spectrometry as nanoESI infusion platform and separation method. Anal Chim Acta. 2016;918:50-59.

183. Dada OO, Zhao Y, Jaya N, Salas-Solano O. High-Resolution Capillary Zone Electrophoresis with Mass Spectrometry Peptide Mapping of Therapeutic Proteins: Peptide Recovery and Post-translational Modification Analysis in Monoclonal Antibodies and Antibody-Drug Conjugates. Anal Chem. 2017;89(21):11236-11242.

184. Belov AM, Zang L, Sebastiano R, Santos MR, Bush DR, Karger BL, et al. Complementary middle-down and intact monoclonal antibody proteoform characterization by capillary zone electrophoresis - mass spectrometry. Electrophoresis. 2018;39(16):2069-2082.

185. Haselberg R, De Vijlder T, Heukers R, Smit MJ, Romijn EP, Somsen GW, et al. Heterogeneity assessment of antibody-derived therapeutics at the intact and middle-up level by low-flow sheathless capillary electrophoresis-mass spectrometry. Anal Chim Acta. 2018;1044:181-190.

186. Giorgetti J, Lechner A, Del Nero E, Beck A, François Y-N, Leize-Wagner E. Intact monoclonal antibodies separation and analysis by sheathless capillary electrophoresismass spectrometry. Eur J Mass Spectrom. 2018;146906671880779.

187. Redman EA, Mellors JS, Starkey JA, Ramsey JM. Characterization of Intact Antibody Drug Conjugate Variants Using Microfluidic Capillary Electrophoresis-Mass Spectrometry. Anal Chem. 2016;88(4):2220-2226.

188. Jooß K, Hühner J, Kiessig S, Moritz B, Neusüß C. Two-dimensional capillary zone electrophoresis-mass spectrometry for the characterization of intact monoclonal 
antibody charge variants, including deamidation products. Anal Bioanal Chem. 2017;409(26):6057-6067.

189. Montealegre C, Neusüß C. Coupling imaged capillary isoelectric focusing with mass spectrometry using a nanoliter valve. Electrophoresis. 2018;39(9-10):1151-1154.

190. Byeon J-J, Park M-H, Shin S-H, Lee B ill, Park $\mathrm{Y}$, Choi J, et al. A single liquid chromatography-quadrupole time-of-flight mass spectrometric method for the quantification of total antibody, antibody-conjugated drug and free payload of antibodydrug conjugates. Biomed Chromatogr. 2018;32(7):e4229.

191. Sugimoto H, Ghosh D, Chen S, Smith MD, Abu-Yousif AO, Qian MG. ImmunocaptureLC/MS-Based Target Engagement Measurement in Tumor Plasma Membrane. Anal Chem. 2018;acs.analchem.8b03726.

192. Dong L, Li C, Locuson C, Chen S, Qian MG. A Two-Step Immunocapture LC/MS/MS Assay for Plasma Stability and Payload Migration Assessment of Cysteine-MaleimideBased Antibody Drug Conjugates. Anal Chem. 2018;90(10):5989-5994.

193. Anami Y, Yamazaki CM, Xiong W, Gui X, Zhang N, An Z, et al. Glutamic acid-valinecitrulline linkers ensure stability and efficacy of antibody-drug conjugates in mice. Nat Commun. 2018;9(1):2512.

194. Leipold DD, Figueroa I, Masih S, Latifi B, Yip V, Shen B-Q, et al. Preclinical pharmacokinetics and pharmacodynamics of DCLL9718A: An antibody-drug conjugate for the treatment of acute myeloid leukemia. MAbs. 2018;1-10.

195. He J, Su D, Ng C, Liu L, Yu SF, Pillow TH, et al. High-Resolution Accurate-Mass Mass Spectrometry Enabling In-Depth Characterization of in Vivo Biotransformations for Intact Antibody-Drug Conjugates. Anal Chem. 2017;89(10):5476-5483.

196. Su D, Kozak KR, Sadowsky J, Yu S-F, Fourie-O'Donohue A, Nelson C, et al. Modulating Antibody-Drug Conjugate Payload Metabolism by Conjugation Site and Linker Modification. Bioconjug Chem. 2018;29(4):1155-1167.

197. Jin W, Burton L, Moore I. LC-HRMS quantitation of intact antibody drug conjugate trastuzumab emtansine from rat plasma. Bioanalysis. 2018;10(11):851-862.

198. Li BT, Shen R, Buonocore D, Olah ZT, Ni A, Ginsberg MS, et al. Ado-Trastuzumab Emtansine for Patients With HER2 -Mutant Lung Cancers: Results From a Phase II Basket Trial. J Clin Oncol. 2018;36(24):2532-2537.

199. Su D, Ng C, Khosraviani M, Yu SF, Cosino E, Kaur S, et al. Custom-designed affinity 
capture LC-MS F(ab')2 assay for biotransformation assessment of site-specific antibody drug conjugates. Anal Chem. 2016;88(23):11340-11346.

200. King GT, Eaton KD, Beagle BR, Zopf CJ, Wong GY, Krupka HI, et al. A phase 1, doseescalation study of PF-06664178, an anti-Trop-2/Aur0101 antibody-drug conjugate in patients with advanced or metastatic solid tumors. Invest New Drugs. 2018;36(5):836847.

201. Shi C, Goldberg S, Lin T, Dudkin V, Widdison W, Harris L, et al. Bioanalytical workflow for novel scaffold protein-drug conjugates: quantitation of total Centyrin protein, conjugated Centyrin and free payload for Centyrin-drug conjugate in plasma and tissue samples using liquid chromatography-tandem mass spectrometry. Bioanalysis. 2018;10(20):1651-1665.

202. Shi C, Goldberg S, Lin T, Dudkin V, Widdison W, Harris L, et al. LC/MS/MS Bioanalysis of Protein-Drug Conjugates-The Importance of Incorporating Succinimide Hydrolysis Products. Anal Chem. 2018;90(8):5314-5321.

203. Excoffier M, Janin-Bussat M-C, Beau-Larvor C, Troncy L, Corvaia N, Beck A, et al. A new anti-human Fc method to capture and analyze ADCs for characterization of drug distribution and the drug-to-antibody ratio in serum from pre-clinical species. $J$ Chromatogr B. 2016;1032:149-154.

204. Li KS, Chu PY, Fourie-O'Donohue A, Srikumar N, Kozak KR, Liu Y, et al. Automated On-tip Affinity Capture Coupled with Mass Spectrometry to Characterize Intact Antibody-Drug Conjugates from Blood. J Am Soc Mass Spectrom. 2018;29(7):15321537.

205. He J, Yu S-F, Yee S, Kaur S, Xu K. Characterization of in vivo biotransformations for trastuzumab emtansine by high-resolution accurate-mass mass spectrometry. MAbs. 2018;1-8.

206. Walles M, Rudolph B, Wolf T, Bourgailh J, Suetterlin M, Moenius T, et al. New Insights in Tissue Distribution, Metabolism, and Excretion of [3H]-Labeled Antibody Maytansinoid Conjugates in Female Tumor-Bearing Nude Rats. Drug Metab Dispos. 2016;44(7):897-910.

207. Lanshoeft C, Stutz G, Elbast W, Wolf T, Walles M, Stoeckli M, et al. Analysis of small molecule antibody-drug conjugate catabolites in rat liver and tumor tissue by liquid extraction surface analysis micro-capillary liquid chromatography/tandem mass spectrometry. Rapid Commun Mass Spectrom. 2016;30(7):823-832. 
208. Kraynov E, Kamath A V., Walles M, Tarcsa E, Deslandes A, lyer RA, et al. Current Approaches for Absorption, Distribution, Metabolism, and Excretion Characterization of Antibody-Drug Conjugates: An Industry White Paper. Drug Metab Dispos. 2016;44(5):617-623.

209. Davis JA, Kagan M, Read J, Walles M, Hatsis P. Immunoprecipitation middle-up LCMS for in vivo drug-to-antibody ratio determination for antibody-drug conjugates. Bioanalysis. 2017;9(20):1535-1549.

210. Walles M, Connor A, HainzI D. ADME and Safety Aspects of Non-cleavable Linkers in Drug Discovery and Development. Curr Top Med Chem. 2018;17(32):3463-3475.

211. Taplin S, Vashisht K, Walles M, Calise D, Kluwe W, Bouchard P, et al. Hepatotoxicity with antibody maytansinoid conjugates: A review of preclinical and clinical findings. $J$ Appl Toxicol. 2018;38(5):600-615.

212. Sun X, Ponte JF, Yoder NC, Laleau R, Coccia J, Lanieri L, et al. Effects of DrugAntibody Ratio on Pharmacokinetics, Biodistribution, Efficacy, and Tolerability of Antibody-Maytansinoid Conjugates. Bioconjug Chem. 2017;28(5):1371-1381.

213. Shadid M, Bowlin S, Bolleddula J. Catabolism of antibody drug conjugates and characterization methods. Bioorg Med Chem. 2017;25(12):2933-2945.

214. Snyder JT, Malinao M-C, Dugal-Tessier J, Atkinson JE, Anand BS, Okada A, et al. Metabolism of an Oxime-Linked Antibody Drug Conjugate, AGS62P1, and Characterization of Its Identified Metabolite. Mol Pharm. 2018;15(6):2384-2390.

215. Stoll D, Danforth J, Zhang K, Beck A. Characterization of therapeutic antibodies and related products by two-dimensional liquid chromatography coupled with UV absorbance and mass spectrometric detection. J Chromatogr B. 2016;1032:51-60.

216. Srzentić K, Nagornov KO, Fornelli L, Lobas AA, Ayoub D, Kozhinov AN, et al. Multiplexed Middle-Down Mass Spectrometry as a Method for Revealing Light and Heavy Chain Connectivity in a Monoclonal Antibody. Anal Chem. 2018;90(21):1252712535.

217. Cazzamalli S, Dal Corso A, Widmayer F, Neri D. Chemically Defined Antibody- and Small Molecule-Drug Conjugates for in Vivo Tumor Targeting Applications: A Comparative Analysis. J Am Chem Soc. 2018;140(5):1617-1621.

218. Wang-Lin S xin, Zhou C, Kamath A V., Hong K, Koppada N, Saad OM, et al. Minimal physiologically-based pharmacokinetic modeling of DSTA4637A, A novel THIOMAB ${ }^{\text {TM }}$ antibody antibiotic conjugate against Staphylococcus aureus, in a mouse model. MAbs. 
2018;1-13.

219. Zhou C, Lehar S, Gutierrez J, Rosenberger CM, Ljumanovic N, Dinoso J, et al. Pharmacokinetics and pharmacodynamics of DSTA4637A: A novel THIOMAB $^{\text {TM }}$ antibody antibiotic conjugate against Staphylococcus aureus in mice. MAbs. 2016;8(8):1612-1619.

220. Ambrogelly A, Gozo S, Katiyar A, Dellatore S, Kune Y, Bhat R, et al. Analytical comparability study of recombinant monoclonal antibody therapeutics. MAbs. 2018;10(4):513-538.

221. Nowak C, K. Cheung J, M. Dellatore S, Katiyar A, Bhat R, Sun J, et al. Forced degradation of recombinant monoclonal antibodies: A practical guide. MAbs. 2017;9(8):1217-1230. 